\title{
Numerical specified homogenization of a discrete model with a local perturbation and application to traffic flow
}

\author{
W. Salazar ${ }^{1}$
}

(Received 30 November 2016; revised 7 April 2018)

\begin{abstract}
We present techniques for the analysis and numerical analysis of non-local non-linear PDEs. We apply these techniques to an equation derived from the modelling of traffic flow. We introduce a macroscopic model in the form of a Hamilton-Jacobi equation with a junction condition. More precisely, the goal of this work is to obtain the numerical homogenization of a non-local PDE deriving from a first order discrete model for traffic flow that simulates the presence of a local perturbation. Previously we showed that the solution of the discrete microscopic model converges to the (unique) solution of a HamiltonJacobi equation posed on a network and with a junction condition (it can be seen as a flux limiter that keeps the memory of the local perturbation). The goal of this article is to provide a numerical scheme
\end{abstract}

DoI:10.21914/anziamj.v59i0.11559, (C) Austral. Mathematical Soc. 2018. Published 2018-05-16. ISSN 1445-8810. (Print two pages per sheet of paper.) Copies of this article must not be made otherwise available on the internet; instead link directly to the DOI for this article. 
able to obtain an approximation of this flux-limiter. We prove the convergence of this scheme and we give some numerical results.

Subject class: 35D40, 90B20, 35B27, 35F20, 45K05, 65M06

Keywords: numerical specified homogenization, Hamilton-Jacobi equations, viscosity solutions, traffic flow, microscopic models, macroscopic models, convergence of numerical scheme

\section{Contents}

1 Introduction

E52

1.1 General model: first order model with a local perturbation E54

1.2 Injecting the system of ODEs into a single PDE . . . . . E55

1.3 Convergence result . . . . . . . . . . . E E56

2 Construction of the flux-limiter

E59

3 Numerical scheme for (9)

E60

3.1 Discretization of $(9) \ldots \ldots \ldots \ldots$ E6 . . . . . . . E

3.2 Viscosity solution for the numerical scheme for the approximated cell problem ................

E63

4 Viscosity solutions for the approximated cell problem

E64

4.1 Viscosity solution for the continuous approximated cell problem

E64

5 Convergence of the numerical scheme for the approximated cell problem

E69

6 Numerical simulations

E73

6.1 The algorithm . . . . . . . . . . . . . E E73

6.2 Setting of the computation . . . . . . . . E E74

6.3 Qualitative properties of $\bar{A} \ldots \ldots \ldots$ E76

6.4 Numerical tests . . . . . . . . . . . . . E76 
6.5 Conclusion . . . . . . . . . . . . . . E86

A Discrete approximated cell problem

E88

A.1 Comparisons for the numerical scheme . . . . . . . E88

A.2 Construction of minimal and maximal solutions ..... . E90

\section{Introduction}

The problem of simulating traffic flow is very important, particularly because it allows us to know how the traffic would react to a change in the infrastructure of the road. Traffic flow can be simulated at different scales: the microscopic scale (which describes the dynamics of all the vehicles); the macroscopic scale (which describes macroscopic quantities such as the vehicle density, the average speed, ....); and the mesoscopic scale (between the microscopic and the macroscopic scale). We only consider the microscopic and the macroscopic scales.

Classic microscopic models describe how each vehicle behaves (in a single road). Moreover, they are very precise, intuitive, and based on solid assumptions. However, if we wish to simulate the traffic at large scales (like an entire city), then we cannot use microscopic models because it would be too expensive from a computational point of view. On the other hand, classic macroscopic models simulate traffic using quantities such as the density of vehicles, and the average velocity. These models are more adapted to simulate traffic at larger scales. However, they often are very hard to manipulate and based on assumptions difficult to verify. This is why it is interesting to rigorously obtain the macroscopic equivalent of microscopic models.

We introduce a new macroscopic model for traffic flow. The difference of this model with the classical Bando model [2] or the ones by Batista and Twrdy [5] is that it takes into account the effect of a local perturbation. To obtain such a model we use in Section 2 the result from Forcadel et al. [13] which 
is an homogenization result of a non-local non-linear PDE and we combine it with the numerical analysis of a non-local non-linear PDE. The numerical scheme we use in Section 3 was inspired by the ones presented by Cacace et al. [6], Costeseque et al. [7], and Forcadel [11] and can be adapted for other non-local non-linear PDEs. Similarly, the algorithm we provide in this article can be adapted (provided the numerical scheme satisfies certain monotonicity conditions) for other non-local non-linear PDEs.

Previously [13] we obtained an incomplete macroscopic model (in the form of a Hamilton-Jacobi equation) equivalent to a microscopic model that simulates the presence of local perturbation that does not depend on time (for instance a school zone, or a moderator). In order to obtain the complete model we need to approximate a constant, called the flux-limiter, that contains the information from the local perturbation but at the macroscopic scale. To be more specific, we are interested in the numerical homogenization of a non-local PDE that derives from a microscopic first order model for traffic flow of the type "follow-the-leader" (see (1)) that simulates the presence of a local perturbation. In Section 6, we present an algorithm that allows us to compute an approximation of the flux-limiter.

Forcadel et al. [13, Theorem 2.8] obtained the homogenization of the PDE and the homogenized system is defined by a function call the effective Hamiltonian and by a constant call the flux limiter. The effective Hamiltonian describes the dynamics of the traffic flow and the flux-limiter defines how the local perturbation affects the macroscopic (homogenized) model. The effective Hamiltonian has been explicitly determined [13, Theorem 2.8]; however, the flux-limiter constant is only implicitly determined. Section 3 provides a numerical scheme for the computation of an approximation of the flux-limiter. 


\subsection{General model: first order model with a local perturbation}

We detail the microscopic model from which derives the PDE we study throughout this article. We consider the following model where all the vehicles are considered as points placed in the real line, for all $t>0$,

$$
\dot{U}_{j}(t)=V\left(U_{j+1}(t)-U_{j}(t)\right) \cdot \phi\left(U_{j}(t)\right) \quad \text { with } j \in \mathbb{Z},
$$

where $\mathrm{U}_{j}:[0,+\infty) \rightarrow \mathbb{R}$ denotes the position of the $j$ th vehicle and $\dot{\mathrm{U}}_{\mathrm{j}}$ is its velocity. The function $\phi: \mathbb{R} \rightarrow[0,1]$ simulates the presence of a local perturbation around the origin. We denote by $r$ the radius of influence of the perturbation.

The function $V$ is called the optimal velocity function and we make the following assumptions on $\mathrm{V}$ and $\phi$.

Assumption 1. 1. $\mathrm{V}: \mathbb{R} \rightarrow \mathbb{R}^{+}$is Lipschitz continuous, non-negative.

2. $V$ is non-decreasing on $\mathbb{R}$.

3. There exists $h_{0} \in(0,+\infty)$ such that for all $h \leqslant h_{0}, V(h)=0$.

4. There exists $h_{\max } \in\left(h_{0},+\infty\right)$ such that for all $h \geqslant h_{\max }, V(h)=$ $\mathrm{V}\left(\mathrm{h}_{\max }\right)=: \mathrm{V}_{\max }$.

5. There exists a real $p_{0} \in\left[-1 / h_{0}, 0\right)$ such that the function $p \mapsto$ $\mathrm{pV}(-1 / \mathrm{p})$ is decreasing on $\left[-1 / h_{0}, \mathrm{p}_{0}\right)$ and increasing on $\left[\mathrm{p}_{0}, 0\right)$.

6. The function $\phi: \mathbb{R} \rightarrow[0,1]$ is Lipschitz continuous and $\phi(x)=1$ for $|x| \geqslant r$.

Remark 2. Assumption 1.1 to Assumption 1.3 and Assumption 1.5 are satisfied by several classical optimal velocity functions. We add Assumption 1.4 to work with $\mathrm{V}^{\prime}$ with a bounded support. But by modifying slightly the classical optimal velocity functions, we obtain a function that satisfies all the assumptions. For instance, in the case of the Greenshields based models [16] 
Figure 1: Schematic representation of the optimal velocity function $V$.

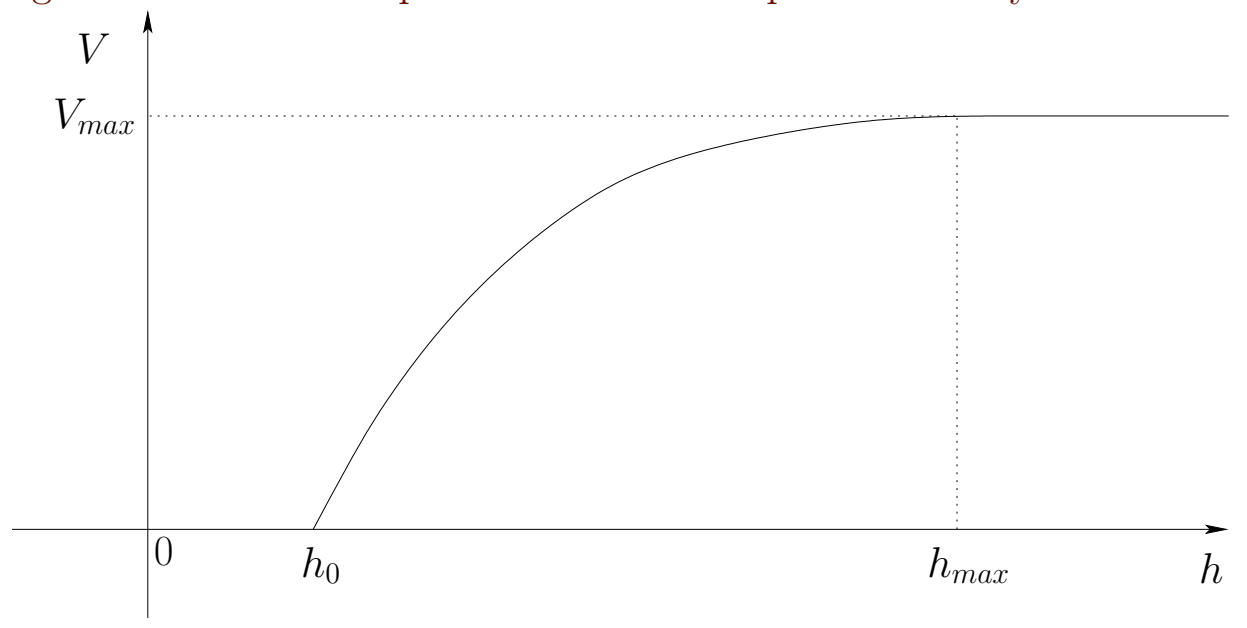

(see also the works of Batista et al. [5], Edie [10], Newell [20] and Garavello et al. [15] for other classical optimal velocity functions):

$$
V(h)= \begin{cases}0 & \text { for } h \leqslant h_{0} \\ V_{\max }\left[1-\left(h_{0} / h\right)^{2}\right] & \text { for } h_{0}<h \leqslant h_{\max } \\ V_{\max }\left[1-\left(h_{0} / h_{\max }\right)^{2}\right] & \text { for } h>h_{\max }\end{cases}
$$

Figure 1 schematically represents an optimal velocity function satisfying Assumption 1.

\subsection{Injecting the system of ODEs into a single PDE}

In order to obtain an homogenization result, I borrowed the idea from Forcadel et al. [12] and injected the system of ODE (1) into a single PDE. To do this, Forcadel et al. [13] introduced the following "cumulative distribution function" 
of vehicles

$$
\rho^{\varepsilon}(t, y)=-\varepsilon\left\{\sum_{i \geqslant 0} H\left[y-\varepsilon U_{i}(t / \varepsilon)\right]+\sum_{i<0}\left[-1+H\left(y-\varepsilon U_{i}(t / \varepsilon)\right)\right]\right\},
$$

with

$$
H(x)= \begin{cases}1 & \text { if } x \geqslant 0 \\ 0 & \text { if } x<0\end{cases}
$$

Forcadel et al. [13] proved that under Assumption 1 the function $\rho^{\varepsilon}$ satisfies in the viscosity sense (it is a weak solution [13]) the following non-local equation for $\mathfrak{u}^{\varepsilon}$ :

$$
\mathfrak{u}_{\mathrm{t}}^{\varepsilon}+\mathrm{M}^{\varepsilon}\left[\frac{\mathrm{u}^{\varepsilon}(\mathrm{t}, \cdot)}{\varepsilon}\right](x) \cdot \phi\left(\frac{\chi}{\varepsilon}\right) \cdot\left|\mathbf{u}_{\chi}^{\varepsilon}\right|=0 \quad \text { on }(0,+\infty) \times \mathbb{R},
$$

where $M^{\varepsilon}$ is a non-local operator defined by

$$
M^{\varepsilon}[G](x)=\int_{-\infty}^{+\infty} J(z) E[G(x+\varepsilon z)-G(x)] d z-\frac{3}{2} V_{\max }
$$

with

$$
\mathrm{E}(z)=\left\{\begin{array}{ll}
0 & \text { if } z \geqslant 0, \\
1 / 2 & \text { if }-1 \leqslant z<0, \\
3 / 2 & \text { if } z<-1,
\end{array} \text { and } \quad J=V^{\prime} \text { on } \mathbb{R} .\right.
$$

We denote by $M$ the operator $M^{\varepsilon}$ with $\varepsilon=1$.

\subsection{Convergence result}

We define $\mathrm{k}_{0}=1 / \mathrm{h}_{0}$ and $\overline{\mathrm{H}}: \mathbb{R} \rightarrow \mathbb{R}$, by

$$
\overline{\mathrm{H}}(p)= \begin{cases}-p-k_{0} & \text { for } p<-k_{0} \\ -V(-1 / p)|p| & \text { for }-k_{0} \leqslant p \leqslant 0 \\ p & \text { for } p>0\end{cases}
$$


Figure 2: Schematic representation of $\overline{\mathrm{H}}$.

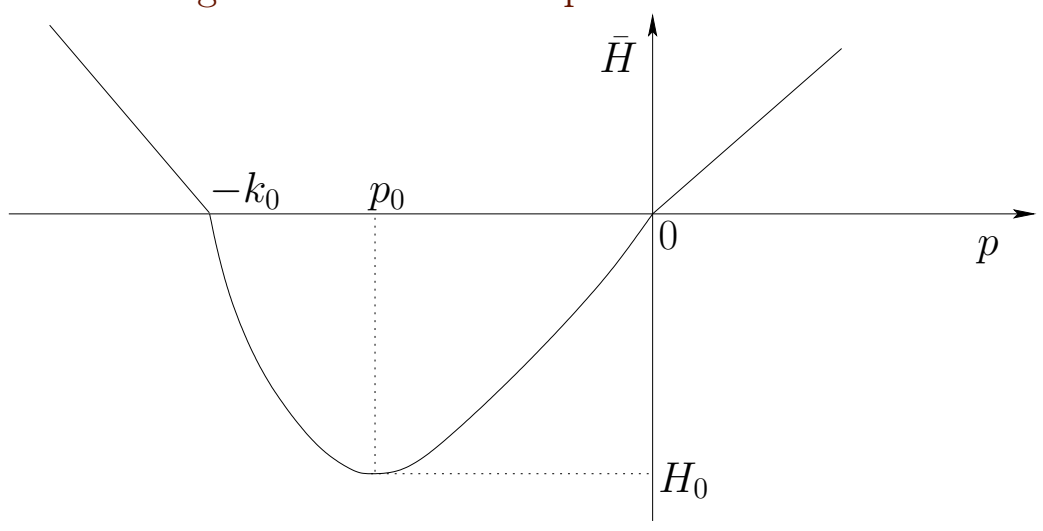

Such an $\bar{H}$ is continuous, coercive $\left(\lim _{|p| \rightarrow+\infty} \bar{H}(p)=+\infty\right)$ and because of Assumption 1.5, there exists a unique point $\mathrm{p}_{0} \in\left[-\mathrm{k}_{0}, 0\right]$ such that

$$
\left\{\begin{array}{l}
\bar{H} \text { is decreasing on }\left(-\infty, p_{0}\right), \\
\bar{H} \text { is increasing on }\left(p_{0},+\infty\right)
\end{array}\right.
$$

We denote by $\mathrm{H}_{0}=\min _{\mathfrak{p} \in \mathbb{R}} \overline{\mathrm{H}}(\mathrm{p})=\overline{\mathrm{H}}\left(\mathrm{p}_{0}\right)$ and Figure 2 shows an schematic representation of $\overline{\mathrm{H}}$.

Forcadel et al. [13] established the following homogenization result.

Theorem 3 (Junction condition by homogenisation). Given Assumption 1, and that at the initial time for all $i \in \mathbb{Z}, \mathrm{U}_{\mathrm{i}}(0) \leqslant \mathrm{U}_{i+1}(0)-\mathrm{h}_{0}$. We also assume that there exists a constant $\mathrm{R}>0$ such that, for all $i \in \mathbb{Z}$, if $\left|\mathrm{U}_{i}(0)\right| \geqslant \mathrm{R}$, then $\mathrm{U}_{\mathrm{i}+1}(0)-\mathrm{U}_{\mathrm{i}}(0)=\mathrm{h}$, with $\mathrm{h} \geqslant \mathrm{h}_{0}$. Then there exists $\bar{A} \in\left[\mathrm{H}_{0}, 0\right]$ such that the function $\rho^{\varepsilon}$ defined by (2) converges towards the 
unique solution $\mathrm{u}^{0}$ of

$$
\begin{cases}u_{t}^{0}+\bar{H}\left(u_{x}^{0}\right)=0 & \text { for }(t, x) \in(0,+\infty) \times(-\infty, 0), \\ u_{t}^{0}+\bar{H}\left(u_{x}^{0}\right)=0 & \text { for }(t, x) \in(0,+\infty) \times(0,+\infty), \\ u_{t}^{0}+F_{\bar{A}}\left(u_{x}^{0}\left(t, 0^{-}\right), u_{x}^{0}\left(t, 0^{+}\right)\right)=0 & \text { for }(t, x) \in(0,+\infty) \times\{0\}, \\ u^{0}(0, x)=-x / h & \text { for } x \in \mathbb{R},\end{cases}
$$

where $\overline{\mathrm{A}}$ has to be determined, and

$$
\mathrm{F}_{\overline{\mathrm{A}}}\left(\mathrm{p}_{-}, \mathrm{p}_{+}\right)=\max \left\{\overline{\mathrm{A}}, \overline{\mathrm{H}}^{+}\left(\mathrm{p}_{-}\right), \overline{\mathrm{H}}^{-}\left(\mathrm{p}_{+}\right)\right\},
$$

with

$$
\overline{\mathrm{H}}^{-}(\mathrm{p})=\left\{\begin{array}{ll}
\overline{\mathrm{H}}(\mathrm{p}) & \text { if } p \leqslant p_{0}, \\
\overline{\mathrm{H}}\left(\mathrm{p}_{0}\right) & \text { if } \mathrm{p} \geqslant \mathrm{p}_{0},
\end{array} \quad \text { and } \quad \overline{\mathrm{H}}^{+}(\mathrm{p})= \begin{cases}\overline{\mathrm{H}}\left(\mathrm{p}_{0}\right) & \text { if } p \leqslant p_{0} \\
\overline{\mathrm{H}}(\mathrm{p}) & \text { if } \mathrm{p} \geqslant \mathrm{p}_{0}\end{cases}\right.
$$

Moreover, $\mathrm{u}^{0}$ satisfies $-\mathrm{k}_{0} \leqslant \mathrm{u}_{\mathrm{x}}^{0} \leqslant 0$.

Link with the macroscopic model LWR There are different types of established macroscopic models. But we focus on the first order model LWR (the Lighthill-Whitham-Richards model [18, 22, e.g.]), which is defined by the following PDE for $\rho$ :

$$
\partial_{t} \rho+\partial_{x}[\rho v(\rho)]=0,
$$

where $\rho(t, x)$ is the density of vehicles at the point $x \in \mathbb{R}$ (physical point on the road) at time $t \in(0,+\infty)$, and $v(\rho)$ is the average speed of vehicles. We call the function $f(\rho)=\rho v(\rho)$ the traffic flux, which is also called the fundamental diagram. The fundamental diagram completely defines equation (8). Different authors have presented fundamental diagrams trying to best adapt the LWR model to real life observations. The book of Garavello and Piccoli [15] gave more details on those models.

Let us formally consider $\rho^{0}=\partial_{x} u^{0}$ where $u^{0}$ is the unique solution of (5). We can see that $\rho^{0}$ formally satisfies the PDE $\partial_{t} \rho^{0}+\partial_{x}\left[\bar{H}\left(\rho^{0}\right)\right]=0$ for $\chi \neq 0$. 
This PDE is equivalent to an LWR model with the fundamental diagram $p V(1 / p)=: \bar{H}(p)$ and $\rho^{0}=\partial_{x} u^{0}$ corresponds to the density of vehicles. The interest in the formulation (5) is that we have a well defined equation at the junction for all times $t \in(0,+\infty)$. Model (5) is an extension on the LWR model since it provides a rigorous junction condition that simulates a local perturbation. Only using the classical LWR model one is forced to use the initial condition to simulate a local perturbation but the effect of the initial condition does not remain over time. Model (5) provides a way to analyse at the macroscopic scale the influence of local perturbations on traffic flow and this is why it is important to have a complete result and to have (at least) a numerical approximation of the flux-limiter $\bar{A}$ that defines the junction conditions in (5).

\section{Construction of the flux-limiter}

Theorem 3 shows that in order to have a complete homogenization result we only need to determine the flux limiter $\bar{A}$. In the rest of the article we focus on obtaining a numerical approximation of the flux limiter. Section 6 gives a few characterizations of $\bar{A}$ described by Forcadel et al. [13].

The flux limiter is the unique constant $\lambda$ such that there exists a solution $w$ of the Hamilton-Jacobi equation $M[w](x) \cdot \phi(x) \cdot\left|w_{x}\right|=\lambda$ for $x \in \mathbb{R}$, with the right slopes at infinity. Forcadel et al. [14, 13] explained these slopes in detail.

In order to construct the corrector for the junction $w$ and $\bar{A}$, Forcadel, Salazar and Zaydan [13] used the idea from Galise, Imbert and Monneau [14], and from Achdou and Tchou [1] and from the lectures of Lions at "College de France" [19], which is to construct the correctors in a truncated domain with good boundary conditions and then to expand the domain.

The effective flux limiter is then obtained in the limit as $l \rightarrow+\infty$, and then $R \rightarrow+\infty$ of $\lambda_{l, R}$ which is the unique constant for which the following 
truncated cell problem admits a solution [13, Prop. 6.4] for $l \in(r,+\infty), r \ll l$ and $r \leqslant R \ll l$, find $\lambda_{l, R}$, such that there exists a solution $w^{l, R}$ of

$$
\begin{cases}G_{R}\left(x,\left[w^{l, R}\right], w_{x}^{l, R}\right)=\lambda_{l, R} & \text { if } x \in(-l, l), \\ \bar{H}^{-}\left(w_{x}^{l, R}\right)=\lambda_{l, R} & \text { if } x=-l, \\ \bar{H}^{+}\left(w_{x}^{l, R}\right)=\lambda_{l, R} & \text { if } x=l,\end{cases}
$$

with $G_{R}(x,[U], q)=\psi_{R}(x) \phi(x) \cdot M[U](x) \cdot|q|+\left(1-\psi_{R}(x)\right) \cdot \bar{H}(q)$, and $\psi_{R} \in C^{\infty}, \psi_{R}: \mathbb{R} \rightarrow[0,1]$, with $\psi_{R}(x)<1$ for all $x \notin[-R, R]$ and

$$
\psi_{R} \equiv \begin{cases}1 & \text { on }[-R, R] \\ 0 & \text { outside }[-R-10, R+10] .\end{cases}
$$

Remark 4 . The operator $\mathrm{G}_{\mathrm{R}}$ is used to have a local operator near the boundary and then to well define the boundary conditions.

To obtain an approximation of $\lambda_{l, R}$ we follow the construction of Forcadel et al. [13] and consider the approximated truncated cell problem, for all $\delta>0$ :

$$
\left\{\begin{array}{cl}
\delta v^{\delta}+\psi_{R}(x) M\left[v^{\delta}\right](x) \cdot \phi(x) \cdot\left|v_{x}^{\delta}\right| & \\
+\left(1-\psi_{R}(x)\right) \bar{H}\left(v_{x}^{\delta}\right)=0 & \text { for } x \in(-l, l), \\
\delta v^{\delta}+\bar{H}^{-}\left(v_{x}^{\delta}\right)=0 & \text { for } x=-l, \\
\delta v^{\delta}+\bar{H}^{+}\left(v_{x}^{\delta}\right)=0 & \text { for } x=l .
\end{array}\right.
$$

Forcadel, Salazar and Zaydan [13] proved that $-\delta \nu^{\delta}(0) \rightarrow \lambda_{\mathrm{l}, \mathrm{R}}$ as $\delta \rightarrow 0$.

\section{$3 \quad$ Numerical scheme for (9)}

\subsection{Discretization of (9)}

The numerical scheme we use was inspired by one from Cacace et al. [6], and from Forcadel [11] for the non-local operator, and by Costeseque et al. [7] 
for the local operator. We consider a uniform grid of the interval $[-l, l]$ with $2 \mathrm{n}+1$ points, $\mathrm{n} \in \mathbb{N} \backslash\{0\}$, and we denote by $\Delta \mathrm{x}=\mathrm{l} / \mathrm{n}$ the discretization step. For all $i \in\{-\mathfrak{n}, \ldots, n\}$, we denote by $x_{i}=\Delta x \cdot i$ the nodes of the grid. In particular $x_{0}=0, x_{-n}=-l$ and $x_{n}=l$.

For every discrete function $v:\{-\mathfrak{n}, \ldots, \mathfrak{n}\} \rightarrow \mathbb{R}$, we denote by $v_{\sharp}$ its piecewise constant extension to $\mathbb{R}$, defined by

$$
v_{\sharp}(x)=\sum_{i=1}^{n} v_{i} \cdot \chi_{Q_{i}}(x)
$$

with

$$
Q_{i}= \begin{cases}{[-l,-l+\Delta x / 2)} & \text { if } i=-n \\ {\left[x_{i}-\Delta x / 2, x_{i}+\Delta x / 2\right)} & \text { if } i \in\{-n+1, \ldots, n-1\} \\ {[l-\Delta x / 2, l]} & \text { if } i=n\end{cases}
$$

Discretization of the non-local operator For all discrete function $v$ : $\{-\mathfrak{n}, \ldots, \mathfrak{n}\} \rightarrow \mathbb{R}$, we define the discrete non-local operators $M_{i}^{\mathrm{d}}[v]:=$ $M\left[v_{\sharp}\right]\left(x_{i}\right)$ and $\tilde{M}_{i}^{\mathrm{d}}[v]:=\tilde{M}\left[v_{\sharp}\right]\left(x_{i}\right)$, where $\tilde{M}$ is defined like $M$, (3) with $\varepsilon=1$, but replacing $\mathrm{E}$ with

$$
\tilde{\mathrm{E}}(z)= \begin{cases}0 & \text { if } z>0 \\ 1 / 2 & \text { if }-1<z \leqslant 0 \\ 3 / 2 & \text { if } z \leqslant-1\end{cases}
$$

Discretization of the gradient We consider the standard forward and backward first order differences:

$$
\mathrm{D}^{+} v\left(\mathrm{x}_{\mathrm{i}}\right)=\frac{v\left(\mathrm{x}_{\mathrm{i}+1}\right)-v\left(\mathrm{x}_{\mathrm{i}}\right)}{\Delta \mathrm{x}} \text { and } \mathrm{D}^{-} v\left(\mathrm{x}_{\mathrm{i}}\right)=\frac{v\left(\mathrm{x}_{\mathrm{i}}\right)-v\left(\mathrm{x}_{\mathrm{i}-1}\right)}{\Delta \mathrm{x}} .
$$

Finally, we consider the two component vector $D v_{i}=\left(D^{-} v\left(x_{i}\right), D^{+} v\left(x_{i}\right)\right)$. 
Similarly to Cacace et al. [6], we consider the following Osher-Sethian [21] upwind discretization of the modulus of the gradient. Let $S=(p, q) \in \mathbb{R}^{2}$, we define the following function, that we use for the discretization of the gradient $\mathrm{G}^{+}(\mathrm{S})=\left[\max (\mathrm{p}, 0)^{2}+\min (\mathbf{q}, 0)^{2}\right]^{1 / 2}$.

Discretization of the local operator Concerning the local operator $\overline{\mathrm{H}}(\cdot)$, as in Costeseque et al. [7] we consider the discretization

$$
\overline{\mathrm{H}}_{\mathrm{d}}\left(\mathrm{D} v_{\mathrm{i}}\right)=\max \left\{\overline{\mathrm{H}}^{+}\left(\mathrm{D}^{-} v_{\mathrm{i}}\right), \overline{\mathrm{H}}^{-}\left(\mathrm{D}^{+} v_{\mathrm{i}}\right)\right\},
$$

where $\overline{\mathrm{H}}^{-}$and $\overline{\mathrm{H}}^{+}$are defined in (7).

Finally, we introduce for any discrete function $v:\{-\mathfrak{n}, \ldots, \mathfrak{n}\} \rightarrow \mathbb{R}$,

$$
\begin{aligned}
R_{i}[v]:=\mathcal{R}_{i}\left([v], D v_{i}\right)= & \psi_{R}\left(x_{i}\right) \cdot M_{i}^{d}[v] \cdot \phi\left(x_{i}\right) \cdot G^{+}\left(D v_{i}\right) \\
& +\left(1-\psi_{R}\left(x_{i}\right)\right) \cdot \bar{H}_{d}\left(D v_{i}\right) .
\end{aligned}
$$

Similarly, we define $\tilde{R}$ and $\tilde{\mathcal{R}}$ by replacing $M^{\mathrm{d}}$ with $\tilde{M}^{\mathrm{d}}$.

To summarize, in the rest of the article, for all discrete function $v:\{-\mathfrak{n}, \ldots, \mathfrak{n}\} \rightarrow$ $\mathbb{R}$, we consider the following numerical scheme:

$$
\begin{cases}\delta v_{i}+R_{i}[v]=0 & \text { for } i \in\{-n+1, \ldots, n-1\} \\ \delta v_{i}+\bar{H}^{-}\left(D^{+} v_{i}\right)=0 & \text { for } i=-n \\ \delta v_{i}+\bar{H}^{+}\left(D^{-} v_{i}\right)=0 & \text { for } i=n\end{cases}
$$

Remark 5 (Notation for the discretization of the non-local operator). Since the function J inside the non-local operator is of bounded support, we introduce the following notations, which are the discrete equivalents of $h_{0}$ and $h_{\max }$,

$$
\begin{aligned}
& j_{0}=\max \left\{j \in\{-\mathfrak{n}, \ldots, \mathfrak{n}\} \text { s.t. } x_{j}-\Delta x / 2<h_{0}\right\}, \\
& j_{\max }=\min \left\{j \in\{-\mathfrak{n}, \ldots, \mathfrak{n}\} \text { s.t. } x_{j}+\Delta x / 2>h_{\max }\right\} \text {. }
\end{aligned}
$$




\subsection{Viscosity solution for the numerical scheme for the approximated cell problem}

To simplify the notation, we introduce

$$
F_{i}\left([v], D v_{i}\right)= \begin{cases}\mathcal{R}_{i}\left([v], D v_{i}\right) & \text { if } x_{i} \in(-l, l) \\ \bar{H}^{-}\left(D^{+} v_{i}\right) & \text { if } x_{i}=-l \\ \bar{H}^{+}\left(D^{-} v_{i}\right) & \text { if } x_{i}=l\end{cases}
$$

Similarly, we define $\tilde{\mathrm{F}}$ by replacing $\mathcal{R}$ with $\tilde{\mathcal{R}}$. We introduce the following definition of viscosity solution for (12).

Definition 6 (Viscosity solution for the approximated cell problem scheme). Let us consider a function $v:\{-\mathfrak{n}, \ldots, \mathfrak{n}\} \rightarrow \mathbb{R}$. We say that $v$ is a subsolution (respectively a super-solution) of (12) if for all $i \in\{-\mathfrak{n}, \ldots, \mathfrak{n}\}$ $\delta v_{i}+F_{i}\left([v], D v_{i}\right) \leqslant 0$ (respectively $\left.\delta v_{i}+\tilde{F}_{i}\left([v], D v_{i}\right) \geqslant 0\right)$. Then we say that $v$ is a solution of (12) if and only if it is a sub- and a super-solution.

Remark 7 . The notion of discrete viscosity solutions is necessary here because of the discontinuity inside the non-local operator. We could not work with a regularised version of $E$ because we do not have a stability result with respect to E. Moreover, in Section 5 and Appendix A (which contain mathematical proofs) the interest of such a definition becomes evident. Appendix A proves the existence of solutions for (12).

The main result of this article is the following convergence result whose proof is postponed until Section 5 .

Theorem 8 (Convergence). Using the same notations as in (10). Let $\left(v_{i}^{\Delta x}\right)_{i \in\{-n, \ldots, n\}}$ be a solution of (12), then the function $v_{\sharp}^{\Delta x}$ (defined as in (10)) converges locally uniformly as $\Delta \mathrm{x} \rightarrow 0$ to the unique continuous viscosity solution of (13).

Remark 9 (Condition on the discretization step). In the rest of the article, we consider that the integer $n$ is big enough ( $\Delta x$ is small enough) so that 
$j_{0}>1$. Given the standard values of $h_{0}$ ( $>2$ metres), this is not a very restrictive condition but it helps to simplify the computations (regarding the monotonicity of the scheme) since for any $i \in\{-\mathfrak{n}, \ldots, \mathfrak{n}\}$ the term $v_{i+1}$ appears only on the gradient in $\mathrm{F}_{i}\left([v], \mathrm{D} v_{i}\right)$.

\section{Viscosity solutions for the approximated cell problem}

In this section, we study (9). In order to simplify the notation, we drop the index $\delta$ in $v^{\delta}$. We also present the definition of viscosity solutions for (9). The user's guide of Crandall, Ishii, Lions [8] and the book of Barles [3] give a good introduction to viscosity solutions.

\subsection{Viscosity solution for the continuous approximated cell problem}

For $l \in(r,+\infty), r \ll l$ and $r \leqslant R \ll l$, we consider the problem

$$
\left\{\begin{array}{cl}
\delta v+\psi_{R}(x) M[v](x) \cdot \phi(x) \cdot\left|v_{x}\right| & \\
+\left(1-\psi_{R}(x)\right) \bar{H}\left(v_{x}\right)=0 & \text { for } x \in(-l, l), \\
\delta v+\bar{H}^{-}\left(v_{x}\right)=0 & \text { for } x=-l, \\
\delta v+\bar{H}^{+}\left(v_{x}\right)=0 & \text { for } x=l,
\end{array}\right.
$$

with $M[G](x)=\int_{h_{0}}^{h_{\max }} J(z) E(G(x+z)-G(x)) d z-\frac{3}{2} V_{\max }$,

$$
E(z)=\left\{\begin{array}{ll}
0 & \text { if } z \geqslant 0, \\
1 / 2 & \text { if }-1 \leqslant z<0, \\
3 / 2 & \text { if } z<-1
\end{array} \quad \text { and } \quad J=V^{\prime} \text { on } \mathbb{R} .\right.
$$


Similarly, we define $\tilde{M}[G](x)=\int_{h_{0}}^{h_{\max }} J(z) \tilde{E}(G(x+z)-G(x)) d z-\frac{3}{2} V_{\max }$, with $\tilde{E}$ defined in (11).

In order to simplify the notations, we introduce the function

$$
F\left(x,[v], v_{x}\right)= \begin{cases}\psi_{R}(x) M[v](x) \cdot \phi(x) \cdot\left|v_{x}\right| & \\ \quad+\left(1-\psi_{R}(x)\right) \bar{H}\left(v_{x}\right) & \text { if } x \in(-l, l), \\ \bar{H}^{-}\left(v_{x}\right) & \text { if } x=-l, \\ \bar{H}^{+}\left(v_{x}\right) & \text { if } x=l .\end{cases}
$$

Similarly, we define $\tilde{\mathrm{F}}$ by replacing $M$ by $\tilde{M}$.

We work with viscosity solutions, and the boundary conditions of (13) be interpreted in the classical sense of viscosity solutions with Neumann boundary conditions. That is why we introduce

$$
\mathcal{J}\left(x,[v], v_{x}\right)=\left\{\begin{array}{cl}
\psi_{\mathrm{R}}(x) \mathrm{M}[v](x) \cdot \phi(x) \cdot\left|v_{x}\right| & \\
+\left(1-\psi_{\mathrm{R}}(x)\right) \overline{\mathrm{H}}\left(v_{x}\right) & \text { if } x \in(-l, l), \\
\min \left(\overline{\mathrm{H}}\left(v_{x}\right), \overline{\mathrm{H}}^{-}\left(v_{x}\right)\right) & \text { if } x=-l, \\
\min \left(\overline{\mathrm{H}}\left(v_{x}\right), \overline{\mathrm{H}}^{+}\left(v_{x}\right)\right) & \text { if } x=l,
\end{array}\right.
$$

and

$$
\tilde{\mathcal{J}}\left(x,[v], v_{x}\right)=\left\{\begin{array}{cl}
\psi_{\mathrm{R}}(x) \tilde{\mathrm{M}}[v](x) \cdot \phi(x) \cdot\left|v_{x}\right| & \\
+\left(1-\psi_{\mathrm{R}}(x)\right) \overline{\mathrm{H}}\left(v_{x}\right) & \text { if } x \in(-l, l), \\
\max \left(\overline{\mathrm{H}}\left(v_{x}\right), \overline{\mathrm{H}}^{-}\left(v_{x}\right)\right) & \text { if } x=-l, \\
\max \left(\overline{\mathrm{H}}\left(v_{x}\right), \overline{\mathrm{H}}^{+}\left(v_{x}\right)\right) & \text { if } x=l .
\end{array}\right.
$$

Definition 10 (Viscosity solution for the continuous approximated cell problem). An upper semi-continuous function (respectively lower semi-continuous) $v:[-l, l] \rightarrow \mathbb{R}$ is a viscosity sub-solution (respectively a super-solution) of (13) in $[-l, l]$, if for all $x \in[-l, l]$ and all $\varphi \in C^{1}([-l, l])$ such that $u-\varphi$ reaches a maximum (respectively a minimum) at the point $x$,

$$
\delta v(x)+\mathcal{J}\left(x,[v], \varphi^{\prime}\right) \leqslant 0 \quad\left(\text { respectively } \delta v(x)+\tilde{\mathcal{T}}\left(x,[v], \varphi^{\prime}\right) \geqslant 0\right)
$$


We say that a function $v$ is a solution of (13) if $v^{*}$ and $v_{*}$ are respectively a sub and a super-solution of (13).

Remark 11. As in Forcadel et al. [13], we use this type of definition in order to have a stability result for the non-local term. Da Lio et al. [9] and Slepčev [23] gave such a definition.

We now give a slightly stronger result than a stability result for the sub and super-solutions of (13) that I use to prove the convergence in Section 5 of the numerical scheme.

Proposition 12 (Stability for (13)). Let $\left(\mathrm{u}_{\mathrm{m}}\right)_{\mathrm{m}}$ be a sequence of measurable functions and let $\overline{\boldsymbol{u}}$ denote $\limsup ^{*} \mathbf{u}_{\mathrm{m}}$ (respectively $\left.\underline{\mathfrak{u}}=\liminf _{*} \mathrm{u}_{\mathrm{m}}\right)$. Let $\left(x_{m}, p_{m}\right) \rightarrow\left(x_{0}, p\right)$ in $[-l, l] \times \mathbb{R}$ be such that $u_{m}\left(x_{m}\right) \rightarrow \bar{u}\left(x_{0}\right)$ (respectively $\left.u_{\mathfrak{m}}\left(x_{m}\right) \rightarrow \underline{u}\left(x_{0}\right)\right)$. Then

$$
\begin{aligned}
& \liminf _{\mathfrak{m} \rightarrow+\infty} \mathrm{F}\left(x_{\mathfrak{m}},\left[u_{\mathfrak{m}}\right], p_{\mathfrak{m}}\right) \geqslant \mathcal{J}\left(x_{0},[\bar{u}], p\right) \\
& \left(\text { respectively } \limsup _{\mathfrak{m} \rightarrow+\infty} \tilde{F}\left(x_{\mathfrak{m}},\left[u_{\mathfrak{m}}\right], p_{\mathfrak{m}}\right) \leqslant \tilde{\mathcal{J}}\left(x_{0},[\underline{u}], p\right)\right) .
\end{aligned}
$$

In order to prove Proposition 12, we use the following lemma proved by Slepčev [23].

Lemma 13. Let $\left(\mathrm{f}_{\mathrm{m}}\right)_{\mathrm{m}}$ be a sequence of measurable functions on $\mathbb{R}$, and consider $\overline{\mathrm{f}}=\lim \sup ^{*} \mathrm{f}_{\mathrm{m}}$ and $\underline{\mathrm{f}}=\liminf _{*} \mathrm{f}_{\mathrm{m}}$. Let $\left(\mathrm{a}_{\mathrm{m}}\right)_{\mathrm{m}}$ be a sequence of $\mathbb{R}$ converging to zero. Then

$$
\begin{aligned}
& \mathcal{L}\left(\left\{\boldsymbol{f}_{\mathrm{m}} \geqslant \mathrm{a}_{\mathrm{m}}\right\} \backslash\{\overline{\mathrm{f}} \geqslant 0\}\right) \rightarrow 0 \quad \text { as } \mathrm{m} \rightarrow+\infty \\
& \text { and } \quad \mathcal{L}\left(\{\underline{\mathrm{f}}>0\} \backslash\left\{\mathrm{f}_{\mathrm{m}}>\mathrm{a}_{\mathrm{m}}\right\}\right) \rightarrow 0 \quad \text { as } \mathrm{m} \rightarrow+\infty,
\end{aligned}
$$

where $\mathcal{L}(A)$ denotes the Lesbegue measure of measurable set $A$.

Proof of Proposition 12: We just do the proof for $\bar{u}$. We distinguish two cases. 
Case 1: $x_{0} \in(-l, l)$. From the definition of $F$, the only problem we have is the non-local operator. We claim that for $\mathrm{m}$ big enough

$$
M\left[u_{m}\right]\left(x_{m}\right) \geqslant M[\bar{u}]\left(x_{0}\right)-\varepsilon_{m} \text { with } \lim _{m \rightarrow+\infty} \varepsilon_{m}=0 .
$$

Using (4), $E(\beta)=\frac{1}{2} 1_{\{\beta \in[-1,0)\}}+\frac{3}{2} 1_{\{\beta<-1\}}=\frac{1}{2} 1_{\{\beta<0\}}+1_{\{\beta<-1\}}$. We get that

$$
\begin{aligned}
& \int_{\mathbb{R}} J(z) E\left(u_{\mathfrak{m}}\left(x_{\mathfrak{m}}+z\right)-u_{\mathfrak{m}}\left(x_{\mathfrak{m}}\right)\right) d z-\int_{\mathbb{R}} J(z) E\left(\bar{u}\left(x_{0}+z\right)-\bar{u}\left(x_{0}\right)\right) d z \\
& =\int_{\mathbb{R}} J(z)\left\{1_{\left\{u_{\mathfrak{m}}\left(x_{\mathfrak{m}}+z\right)-u_{\mathfrak{m}}\left(x_{\mathfrak{m}}\right)<-1\right\}}-1_{\left\{\bar{u}\left(x_{0}+z\right)-\bar{u}\left(x_{0}\right)<-1\right\}}\right\} d z \\
& \quad+\int_{\mathbb{R}} \frac{1}{2} J(z)\left\{1_{\left\{u_{\mathfrak{m}}\left(x_{\mathfrak{m}}+z\right)-u_{\mathfrak{m}}\left(x_{\mathfrak{m}}\right)<0\right\}}-1_{\left\{\bar{u}\left(x_{0}+z\right)-\bar{u}\left(x_{0}\right)<0\right\}}\right\} d z .
\end{aligned}
$$

Using Lemma 13, for $\mathrm{n}$ big enough,

$$
\begin{gathered}
\int_{\mathbb{R}} J(z)\left\{1_{\left\{\bar{u}\left(x_{0}+z\right)-\bar{u}\left(x_{0}\right) \geqslant-1\right\}}-1_{\left\{u_{\mathfrak{m}}\left(x_{\mathfrak{m}}+z\right)-u_{\mathfrak{m}}\left(x_{\mathfrak{m}}\right) \geqslant-1\right\}}\right\} d z \\
\geqslant-\int_{\mathbb{R}} J(z) 1_{\left\{A_{\mathfrak{m}}(z) \backslash A(z)\right\}} \geqslant-\frac{\varepsilon_{\mathfrak{m}}}{2}, \\
\int_{\mathbb{R}} \frac{1}{2} J(z)\left\{1_{\left\{\bar{u}\left(x_{0}+z\right)-\bar{u}\left(x_{0}\right) \geqslant 0\right\}}-1_{\left\{u_{\mathfrak{m}}\left(x_{\mathfrak{m}}+z\right)-u_{\mathfrak{m}}\left(x_{\mathfrak{m}}\right) \geqslant 0\right\}}\right\} d z \\
\geqslant-\frac{1}{2} \int_{\mathbb{R}} J(z) 1_{\left\{B_{\mathfrak{m}}(z) \backslash \mathrm{B}(z)\right\}} \geqslant-\frac{\varepsilon_{\mathfrak{m}}}{2},
\end{gathered}
$$

with

$$
\left\{\begin{array}{l}
A_{\mathfrak{m}}(z)=\left\{u_{\mathfrak{m}}\left(x_{\mathfrak{m}}+z\right)-u_{\mathfrak{m}}\left(x_{\mathfrak{m}}\right) \geqslant-1\right\} \cup\left\{\bar{u}\left(x_{0}+z\right)-\bar{u}\left(x_{0}\right) \geqslant-1\right\} \\
A(z)=\left\{\bar{u}\left(x_{0}+z\right)-\bar{u}\left(x_{0}\right) \geqslant-1\right\}
\end{array}\right.
$$

and

$$
\left\{\begin{array}{l}
B_{m}(z)=\left\{u_{m}\left(x_{m}+z\right)-u_{m}\left(x_{m}\right) \geqslant 0\right\} \cup\left\{\bar{u}\left(x_{0}+z\right)-\bar{u}\left(x_{0}\right) \geqslant 0\right\} \\
B(z)=\left\{\bar{u}\left(x_{0}+z\right)-\bar{u}\left(x_{0}\right) \geqslant 0\right\} .
\end{array}\right.
$$


Using (16) and (17), we prove (15). Given that $x_{0} \in(-l, l)$, for $m$ big enough we have $x_{m} \in(-l, l)$. Using the definition of $F$ and (15),

$$
\begin{aligned}
& \psi_{R}\left(x_{m}\right) M\left[u_{m}\right]\left(x_{m}\right) \cdot \phi\left(x_{m}\right) \cdot\left|p_{m}\right|+\left(1-\psi_{R}\left(x_{m}\right)\right) \bar{H}\left(p_{m}\right) \\
& \geqslant \\
& \quad \psi_{R}\left(x_{m}\right) M[\bar{u}]\left(x_{0}\right) \cdot \phi\left(x_{m}\right) \cdot\left|p_{m}\right| \\
& \quad+\left(1-\psi_{R}\left(x_{m}\right)\right) \bar{H}\left(p_{m}\right)-\varepsilon_{m} \psi_{R}\left(x_{m}\right) \cdot \phi\left(x_{m}\right) \cdot\left|p_{m}\right| .
\end{aligned}
$$

Using that the terms on the right are continuous, we pass to the limit as $m$ goes to infinity to obtain (14).

Case 2: $x_{0}=-l$ or $x_{0}=l$. In this case, using Definition 10 and the continuity of $\overline{\mathrm{H}}$, we obtain (14). This ends the proof of Proposition 12.

Theorem 14 (Comparison principle). Let $\boldsymbol{u}$ and $v$ be respectively a sub and a super-solution of (13), then for all $x \in[-l, l], \mathfrak{u}(x) \leqslant v(x)$.

Proof: The proof of this theorem comes from the comparison principle [12, Thm 4.4] for the non-local term. The only remaining difficulty is proving this result at the boundaries. However, for $x$ close to $l_{2}$ the function $G_{R}$ is the effective Hamiltonian $\bar{H}$. Therefore, we proceed as in the proof of Galise et al. [14, Prop. 4.1] and consider the boundaries as a network composed of a single lane and a junction point then we use the results from Imbert and Monneau [17] and so we skip the rest of the proof. Notice that Definition 10 is equivalent at the boundaries to the definition of relaxed viscosity solution [17, Defn 2.1] in the case of a single lane with a junction point.

Theorem 15 (Existence and uniqueness). Given Assumption 1, there exists a unique solution $v^{\delta}$ of (13). Moreover, this solution is continuous and for all $x \in[-l, l], 0 \leqslant v^{\delta}(x) \leqslant C_{0} / \delta$. 
Finally, the following result from Forcadel et al. [13, Proof of Prop. 6.4] justifies considering (13) and looking for a numerical approximation of $v^{\delta}$.

Proposition 16. Up to a subsequence, $\lim _{\delta \rightarrow 0}-\delta v^{\delta}(0)=\lambda_{l, R}$.

Remark 17 (Bounds on the non-local operator). Given the definition of the non-local operators $M$ and $\tilde{M}$, for any function $G: \mathbb{R} \rightarrow \mathbb{R}$ and any $x \in \mathbb{R}$, $-\frac{3}{2} \mathrm{~V}_{\max } \leqslant \mathrm{M}[\mathrm{G}](\mathrm{x}) \leqslant \tilde{M}[\mathrm{G}](\mathrm{x}) \leqslant 0$. Given the definition of $\mathrm{M}^{\mathrm{d}}$ and $\tilde{M}^{\mathrm{d}}$, this inequality hold for the discrete non-local operators.

\section{Convergence of the numerical scheme for the approximated cell problem}

This section is devoted to the proof of Theorem 8 which is an adaptation of the proof of convergence from Barles and Souganidis [4] to a non-local PDE. Before passing to the proof of Theorem 8 , we give some preliminary results concerning the monotonicity of the numerical scheme (12) and the discrete barriers of the solutions of (12).

Lemma 18 (Monotonicity of $\tilde{\mathrm{F}}$ and $\mathrm{F}$ ). Consider Assumption 1. Let $v, w$ be two discrete functions such that

$$
v_{j} \leqslant w_{j} \quad \text { for all } j \in \mathbb{Z} .
$$

Also assume that there exists an index $i \in \mathbb{Z}$ such that $v_{i}=w_{i}$. Then $\mathrm{F}_{i}\left([v], \mathrm{D} v_{i}\right) \geqslant \mathrm{F}_{i}\left([w], \mathrm{D} w_{i}\right)$ and $\tilde{\mathrm{F}}_{i}\left([v], \mathrm{D} v_{\mathrm{i}}\right) \geqslant \mathrm{F}_{\mathrm{i}}\left([w], \mathrm{D} w_{\mathrm{i}}\right)$.

Proof: We present the proof for $\tilde{F}_{i}$ and we skip it for $F_{i}$ since the proof is similar. Let us begin by proving the monotonicity for the non-local term, first we want to prove that $\tilde{M}_{i}^{\mathrm{d}}[v] \geqslant \tilde{M}_{i}^{\mathrm{d}}[w]$. Using the notations from Remark 5

$$
\tilde{M}^{d}\left[v_{\sharp}\right]\left(x_{i}\right)=\int_{h_{0}}^{h_{\max }} J(z) E^{*}\left(v_{\sharp}\left(x_{i}+z\right)-v_{\sharp}\left(x_{i}\right)\right) d z-\frac{3}{2} V_{\max }
$$


5 Convergence of the numerical scheme for the approximated cell problemE70

$$
\begin{aligned}
& =\sum_{j=j_{0}}^{j_{\text {max }}} \int_{x_{j}-\Delta x / 2}^{x_{i}+\Delta x / 2} J(z) E^{*}\left(v_{\sharp}\left(x_{i}+z\right)-v_{\sharp}\left(x_{j}\right)\right) d z-\frac{3}{2} V_{\text {max }} \\
& =\sum_{j=j_{0}}^{j_{\text {max }}} \int_{Q_{j}} J(z) d z\left\{E^{*}\left(v_{i+j}-v_{i}\right)\right\}-\frac{3}{2} V_{\text {max }} \\
& \geqslant \sum_{j=j_{0}}^{j_{\text {max }}} \int_{Q_{j}} J(z) d z\left\{E^{*}\left(w_{i+j}-w_{i}\right)\right\}-\frac{3}{2} V_{\text {max }}=\tilde{M}_{i}^{d}[w],
\end{aligned}
$$

where we used for the last line that $\tilde{\mathrm{E}}$ is non-increasing, with (18) and that J is non-negative.

Moreover, using (18) $\mathrm{D}^{+} \boldsymbol{v}_{\mathrm{i}} \leqslant \mathrm{D}^{+} \boldsymbol{w}_{\boldsymbol{i}}$ and $\mathrm{D}^{-} \boldsymbol{v}_{\mathrm{i}} \geqslant \mathrm{D}^{-} \boldsymbol{w}_{\boldsymbol{i}}$. These imply in particular that both $\max \left(\mathrm{D}^{+} \boldsymbol{v}_{\boldsymbol{i}}, 0\right)^{2} \leqslant \max \left(\mathrm{D}^{+} \boldsymbol{w}_{i}, 0\right)^{2}$ and $\min \left(\mathrm{D}^{-} \boldsymbol{v}_{i}, 0\right)^{2} \leqslant$ $\min \left(\mathrm{D}^{-} \boldsymbol{w}_{i}, 0\right)^{2}$. Combining the previous inequalities gives the inequality $\mathrm{G}^{+}\left(\mathrm{D} v_{\mathrm{i}}\right) \leqslant \mathrm{G}^{+}\left(\mathrm{D} \boldsymbol{w}_{\mathrm{i}}\right)$. Recall that $\tilde{\mathrm{M}}^{\mathrm{d}}$ is non-positive (Remark 17) and therefore,

$$
\tilde{M}_{i}^{\mathrm{d}}[v] \cdot \mathrm{G}^{+}\left(D v_{i}\right) \geqslant \tilde{M}_{i}^{\mathrm{d}}[v] \cdot \mathrm{G}^{+}\left(D w_{i}\right) \geqslant \tilde{M}_{i}^{\mathrm{d}}[w] \cdot \mathrm{G}^{+}\left(D w_{i}\right) .
$$

Let us now prove the monotonicity for the local term, using that $\overline{\mathrm{H}}^{+}$is nondecreasing and that $\overline{\mathrm{H}}^{-}$is non-increasing, since both $\overline{\mathrm{H}}^{+}\left(\mathrm{D}^{-} \boldsymbol{v}_{\mathrm{i}}\right) \geqslant \overline{\mathrm{H}}^{+}\left(\mathrm{D}^{-} \mathcal{w}_{\mathrm{i}}\right)$ and $\overline{\mathrm{H}}^{-}\left(\mathrm{D}^{+} \boldsymbol{v}_{\mathrm{i}}\right) \geqslant \overline{\mathrm{H}}^{-}\left(\mathrm{D}^{+} \boldsymbol{w}_{\mathrm{i}}\right)$, this implies in particular that

$$
\begin{aligned}
\overline{\mathrm{H}}^{\mathrm{d}}\left(\mathrm{D} \boldsymbol{v}_{\mathrm{i}}\right) & =\max \left(\overline{\mathrm{H}}^{-}\left(\mathrm{D}^{+} \boldsymbol{v}_{\mathrm{i}}\right), \overline{\mathrm{H}}^{+}\left(\mathrm{D}^{-} \boldsymbol{v}_{\boldsymbol{i}}\right)\right) \\
& \geqslant \max \left(\overline{\mathrm{H}}^{-}\left(\mathrm{D}^{+} \boldsymbol{w}_{\boldsymbol{i}}\right), \overline{\mathrm{H}}^{+}\left(\mathrm{D}^{-} \boldsymbol{w}_{\boldsymbol{i}}\right)\right)=\overline{\mathrm{H}}\left(\mathrm{D} \boldsymbol{w}_{\boldsymbol{i}}\right) .
\end{aligned}
$$

Combining (19) and (20), we get $\tilde{\mathrm{F}}_{i}\left([\boldsymbol{v}], \mathrm{D} \boldsymbol{v}_{\mathrm{i}}\right) \geqslant \tilde{\mathrm{F}}_{\boldsymbol{i}}\left([\boldsymbol{w}], \mathrm{D} \boldsymbol{w}_{\mathrm{i}}\right)$.

Lemma 19 (Existence of discrete barriers for (13)). Consider Assumption 1. The discrete functions defined by $v_{i}^{-}=0$ and $v_{i}^{+}=\mathrm{C}_{0} / \delta$ for all $\boldsymbol{i} \in\{-\mathfrak{n}, \ldots, \mathfrak{n}\}$ with $\mathrm{C}_{0}=\left|\mathrm{H}_{0}\right|=\left|\min _{\mathfrak{p} \in \mathbb{R}} \overline{\mathrm{H}}(\mathrm{p})\right|$ are respectively a sub and a super-solution of (13). 
Proof: We only prove that $v^{+}$is a super-solution, since the sub-solution case is similar and even simpler. Using the form of (13), for all $\boldsymbol{i} \in\{-\mathfrak{n}, \ldots, \mathfrak{n}\}$ $\delta v_{i}^{+}+\tilde{F}_{i}\left(\left[v^{+}\right],(0,0)\right) \geqslant \delta v_{i}^{+}+\mathrm{H}_{0}=\left|\mathrm{H}_{0}\right|+\mathrm{H}_{0}=0$. Therefore, $v^{+}$is a supersolution of (13).

Proposition 20 (Discrete barriers). Let $u_{i}^{-}=0$ and $v_{i}^{+}=C_{0} / \delta$ for all $i \in\{-\mathfrak{n}, \ldots, \mathfrak{n}\}$ with $\mathrm{C}_{0}=\left|\mathrm{H}_{0}\right|$. Then every solution $\boldsymbol{v}$ of (12) satisfies $\mathrm{u}^{-} \leqslant v \leqslant v^{+}$.

Proof: First prove $v-v^{+} \leqslant 0$. We introduce $M=\max _{i \in\{-n, \ldots, n\}}\left\{v_{i}-v_{i}^{+}\right\}$, and we assume the maximum is reached for an index $\mathfrak{i}_{0} \in\{-\mathfrak{n}, \ldots, \mathfrak{n}\}$. Therefore

$$
v_{i_{0}}-M=v_{i_{0}}^{+} \quad \text { and } \quad \tilde{v}_{i}:=v_{i}-M \leqslant v_{i}^{+} \text {for all } i \in\{-n, \ldots, n\} .
$$

Notice that $F_{i}\left([v], D v_{i}\right)$ is invariant by addition of constant to $v$ and therefore, $F_{i}\left([\tilde{v}], D \tilde{v}_{i}\right)=F_{i}\left([v], D v_{i}\right)$. Moreover, using Lemma 18 and (21), $\mathrm{F}_{\mathrm{i}_{0}}\left([v], \mathrm{D} v_{\mathrm{i}_{0}}\right)=\mathrm{F}_{\mathrm{i}_{0}}\left([\tilde{v}], \mathrm{D} \tilde{v}_{i_{0}}\right) \geqslant \mathrm{F}_{\mathrm{i}_{0}}\left(\left[v^{+}\right], \mathrm{D} v_{i_{0}}^{+}\right)$. Using that $v$ is a solution of (12) and in particular a sub-solution, $0 \geqslant \delta v_{i_{0}}+\mathrm{F}_{\mathrm{i}_{0}}\left([\boldsymbol{v}], \mathrm{D} \boldsymbol{v}_{\mathrm{i}_{0}}\right) \geqslant$ $\delta v_{i_{0}}^{+}+\delta M+F_{i_{0}}\left(\left[v^{+}\right], D v_{i_{0}}^{+}\right)$. In particular, replacing $v_{i_{0}}^{+}=C_{0} / \delta$

$$
\delta M \leqslant-C_{0}-F_{i_{0}}\left(\left[v^{+}\right], D v_{i_{0}}^{+}\right)= \begin{cases}0 & \text { if } i_{0}=-\mathfrak{n}, \\ -C_{0} & \text { if } i_{0} \in\{-\mathfrak{n}+1, \ldots, n\},\end{cases}
$$

where we use for the equality the definition of $\mathrm{F}$ and that $\mathrm{D} v_{\mathrm{i}_{0}}^{+}=(0,0)$ (in this case the only term that is not equal to 0 is $\left.\overline{\mathrm{H}}^{-}(0)=\mathrm{H}_{0}=-\mathrm{C}_{0}\right)$. Therefore, $M \leqslant 0$. The proof that $v^{-}-v \leqslant 0$ is similar to the previous one and we skip it.

Proof of Theorem 8: We introduce

$$
\bar{v}(x)=\limsup _{\substack{y \rightarrow x \\ \Delta x \rightarrow 0}} v_{\sharp}^{\Delta x}(y) \quad \text { and } \quad \underline{v}(x)=\liminf _{\substack{y \rightarrow x \\ \Delta x \rightarrow 0}} v_{\sharp}^{\Delta x}(y) .
$$


Here we add the superscript $\Delta x$ in order for the proof to be clearer. As in (10) the function $v_{\sharp}^{\Delta x}$ is the piecewise extension of a discrete function $v^{\Delta x}$ which is a solution of (12) (Definition 6).

We want to prove that $\bar{v}$ and $\underline{v}$ are respectively a sub and a super-solution of (13). If that is true, then the comparison principle for (13) implies that $\bar{v} \leqslant \underline{v}$ on $[-l, l]$. However, by construction $\underline{v} \leqslant \bar{v}$, which implies that $\underline{v}=\bar{v}=v^{\delta}$ the unique continuous solution of (13), this implies the local uniform convergence of $v_{\sharp}^{\Delta x}$.

Let us now prove that $\bar{v}$ is a sub-solution of (13). We only do the proof in the sub-solution case, since the super-solution case is very similar and we skip it. We argue by contradiction and assume that there exists a function $\varphi \in \mathrm{C}^{1}([-l, l])$ and a point $\overline{\mathrm{x}} \in[-l, l]$ such that $\overline{\mathrm{u}}-\varphi$ reaches a strict local maximum at $\bar{x}$ and that $\delta \bar{v}(\bar{x})+\mathcal{J}\left(\bar{x},[\bar{v}], \varphi_{x}(\bar{x})\right)=\theta>0$. Moreover, without any loss of generality, we assume that $\bar{u}(\bar{x})=\varphi(\bar{x})$ and that $\varphi \geqslant$ $2 \sup _{\Delta x}\left\|v_{\sharp}^{\Delta x}\right\|_{\infty}$ outside the ball $B(\bar{x}, r)$, where $r>0$ is such that $\bar{u}(x)-\varphi(x) \leqslant$ $0=\bar{u}(\bar{x})-\varphi(\bar{x})$ in $B(\bar{x}, r)$. Then there exists sequences $\Delta x_{m} \in[0,+\infty)$ and $y_{m} \in[-l, l]$, such that as $m \rightarrow+\infty$

$$
\Delta x_{\mathrm{m}} \rightarrow 0, \quad y_{\mathrm{m}} \rightarrow \bar{x}, \quad v_{\sharp}^{\Delta x_{\mathrm{m}}}\left(y_{\mathrm{m}}\right) \rightarrow \bar{v}(\bar{x}), \text { and }
$$

$y_{m}$ is a global maximum point of $v_{\sharp}^{\Delta x_{\mathfrak{m}}}(\cdot)-\varphi(\cdot)$.

We denote by $\xi_{m}=v_{\sharp}^{\Delta x_{m}}\left(y_{m}\right)-\varphi\left(y_{m}\right)$, and we have that $\xi_{m} \rightarrow 0$ as $m \rightarrow$ $+\infty$. Moreover, for all $x \in[-l, l] v_{\sharp}^{\Delta x_{m}}(x) \leqslant \varphi(x)+\xi_{m}$ and $v_{\sharp}^{\Delta x_{m}}\left(y_{m}\right)=$ $\varphi\left(y_{m}\right)+\xi_{m}$. We denote by $x_{i_{m}}$ the point in the grid such that $y_{m} \in Q_{i_{m}}$, therefore $\left|x_{i_{m}}-y_{m}\right| \leqslant \Delta x_{m}$ and

$$
\begin{aligned}
0 & \geqslant \delta v_{i_{m}}^{\Delta x_{m}}+F_{i_{m}}\left(\left[v^{\Delta x_{m}}\right], D v_{i_{m}}^{\Delta x_{m}}\right) \\
& \geqslant \delta v_{i_{m}}^{\Delta x_{m}}+F_{i_{m}}\left(\left[v^{\Delta x_{m}}\right], D \varphi\left(y_{m}\right)\right) \\
& \geqslant \delta v_{\sharp}^{\Delta x_{m}}\left(y_{m}\right)+F\left(x_{i_{m}},\left[v_{\sharp}^{\Delta x_{m}}\right], \varphi^{\prime}\left(x_{0}\right)+o\left(\Delta x_{m}\right)\right),
\end{aligned}
$$

where we used that $v^{\Delta x_{m}}$ is a sub-solution of (12) for the first line. For the 
second line,

$$
\mathrm{D} \varphi\left(\mathrm{y}_{\mathrm{m}}\right)=\left(\frac{\varphi\left(\mathrm{y}_{\mathrm{m}}\right)-\varphi\left(\mathrm{y}_{\mathrm{m}}-\Delta \mathrm{x}_{\mathrm{m}}\right)}{\Delta \mathrm{x}_{\mathrm{m}}}, \frac{\varphi\left(\mathrm{y}_{\mathrm{m}}+\Delta \mathrm{x}_{\mathrm{m}}\right)-\varphi\left(\mathrm{y}_{\mathrm{m}}\right)}{\Delta \mathrm{x}_{\mathrm{m}}}\right)
$$

and invoked the monotonicity of the discrete operator $F_{i_{m}}$ (Lemma 18). Finally, for the third line, we use that $\varphi \in \mathrm{C}^{1}$ and the definition of $\mathrm{F}_{i_{m}}$. We now pass to the limit in the previous inequality

$$
\begin{aligned}
0 & \geqslant \liminf _{\mathfrak{m} \rightarrow+\infty}\left(\delta v_{\sharp}^{\Delta x_{\mathfrak{m}}}\left(i_{m}\right)+\mathrm{F}\left(x_{i_{\mathfrak{m}}},\left[v_{\sharp}^{\Delta x_{\mathfrak{m}}}\right], \varphi^{\prime}\left(x_{0}\right)+o\left(\Delta x_{m}\right)\right)\right) \\
& \geqslant \delta \bar{v}(\bar{x})+\mathcal{J}\left(\bar{x},[\bar{v}], \varphi^{\prime}(\bar{x})\right),
\end{aligned}
$$

where we use Proposition 12 with $u_{m}=v_{\sharp}^{\Delta x_{m}}, x_{m}:=x_{i_{m}}, p_{m}:=\varphi^{\prime}(\bar{x})+$ $\mathrm{o}\left(\Delta \mathrm{x}_{\mathrm{m}}\right)$ and notice that $v_{\sharp}^{\Delta x_{\mathfrak{m}}}\left(x_{i_{\mathfrak{m}}}\right)=v_{\sharp}^{\Delta x_{\mathfrak{m}}}\left(y_{\mathfrak{m}}\right)$. The last inequality provides us with a contradiction which ends the proof of Theorem 8 .

\section{$6 \quad$ Numerical simulations}

In this section, we present an algorithm in Section 6.1 that provides and approximation of the solution of (9) which in turn provides an approximation of the flux-limiter $\bar{A}$. This algorithm comes from the results in Appendix A, and it is based on the properties of the non-local operator. We provide some numerical tests.

\subsection{The algorithm}

The following algorithm is inspired by the one from Cacace et al. [6], and by the results from Appendix A. The idea of the algorithm is to build the extremal solutions from Corollary 30, to build the biggest sub-solution and the smallest super-solution. Therefore obtaining an interval that contains all 
the solutions of (12) and therefore obtaining an approximation of the solution of (13).

We introduce two parameters $\varepsilon_{\mathrm{d}}$ and $\varepsilon_{\mathrm{c}}$ respectively a tolerance to quit the dichotomy process updating the sub and super-solutions (numerical equivalent of Proposition 28) and a tolerance for the convergence of the numerical scheme.

1. Initialization: for $i=-n, \ldots, n, u_{i}^{-}=0$ and $v_{i}^{+}=C_{0} / \delta$.

2. Initialize dichotomy intervals: for $i=-n, \ldots, n, s_{\text {left }, i}^{-}=u_{i}^{-}$and $s_{\text {right }, i}^{-}=u_{i}^{-}+0.1 k_{i}^{-}$with $k_{i}^{-}$the first integer such that $\delta s_{\text {right }, i}^{-}+$ $\mathrm{F}_{i}\left[\mathrm{u}^{-}\right]\left(\mathrm{s}_{\text {right }, i}^{-}\right)>0$, and $s_{\text {left }, i}^{-}=v_{i}^{+}-0.1 k_{i}^{+}$and $s_{\text {right }, i}^{+}=v_{i}^{+}$with $k_{i}^{+}$ the first integer such that $\delta s_{\text {left }, i}^{-}+\tilde{F}_{i}\left[v^{+}\right]\left(s_{\text {left }, i}^{+}\right)<0$.

3. Dichotomy process: for $\mathfrak{i}=-\mathfrak{n}, \ldots, \mathfrak{n}$ optimize respectively in $s_{\mathfrak{i}}^{-} \in$ $\left[\mathrm{s}_{\text {left }, i}^{-}, \mathrm{s}_{\text {right }, i}^{-}\right]$and $s_{i}^{+} \in\left[\mathrm{s}_{\text {left }, i}^{+}, s_{\text {right }, i}^{+}\right]$, the inequalities $\delta s_{i}^{-}+\mathrm{F}_{i}\left[\mathrm{u}^{-}\right]\left(\mathrm{s}_{\mathrm{i}}^{-}\right) \leqslant$ 0 and $\delta s_{i}^{+}+\tilde{\mathrm{F}}_{i}\left[v^{+}\right]\left(s_{i}^{+}\right) \geqslant 0$ until $s_{\text {right }, i}^{-}-s_{\text {left }, i}^{-}<\varepsilon_{d}$ and $s_{\text {right }, i}^{+}-s_{\text {left }, i}^{+}<$ $\varepsilon_{\mathrm{d}}$.

If $\left\|\mathfrak{u}^{-}-s_{\text {left }}^{-}\right\|_{\infty} \leqslant \varepsilon_{\mathrm{c}}$ and $\left\|v^{+}-s_{\text {left }}^{+}\right\|_{\infty} \leqslant \varepsilon_{\mathrm{c}}$ go to Step 4 else swap $\mathrm{u}^{-} \leftrightarrow \mathrm{s}_{\text {left }}^{-}$and $v^{+} \leftrightarrow \mathrm{s}_{\text {right }}^{+}$and go to Step 2 .

4. The interval $\left[\mathbf{u}^{-}, v^{+}\right]$contains all solutions of the numerical scheme (12) and therefore gives an approximation of the solution of (13). In particular, the value of $\bar{A}$ is approximated by the interval $\left[-\delta v_{0}^{+},-\delta u_{0}^{-}\right]$.

Remark 21. This algorithm can be extremely costly computationally. However, parallel programming of Step 2 and Step 3 accelerates the process.

\subsection{Setting of the computation}

We consider an uniform grid of the interval $[-l, l]$ with $2 \mathfrak{n}+1$ points, $\mathfrak{n} \in \mathbb{N} \backslash\{0\}$, and we denote by $\Delta x=l / n$ the discretization step. For all $i \in\{-\mathfrak{n}, \ldots, n\}$, we denote by $x_{i}=\Delta x \cdot i$ the nodes of the grid. 
For all the numerical computations, we consider for equation (13), the following values for the different parameters.

$$
\mathrm{l}=200, \quad \mathrm{R}=100, \quad \varepsilon_{\mathrm{c}}=\varepsilon_{\mathrm{d}}=0.001, \quad \text { and } \delta=0.001 .
$$

For all the numerical computations, regarding the values for the discretization, we consider $n=400$ and $\Delta x=0.5$.

For the computation of the discrete non-local operator, we recall the following result, using the notations from Remark 5 and from (10),

$$
\begin{aligned}
M\left[v_{\sharp}\right]\left(x_{i}\right) & =\int_{j_{0}}^{h_{\max }} J(z) E\left(v_{\sharp}\left(x_{i}+z\right)-v_{\sharp}\left(x_{i}\right)\right) d z-\frac{3}{2} V_{\max } \\
& =\sum_{j=j_{0}}^{j_{\max }} \int_{x_{j}-\Delta x / 2}^{x_{j}+\Delta x / 2} J(z) E\left(v_{\sharp}\left(x_{i}+z\right)-v_{\sharp}\left(x_{j}\right)\right) d z-\frac{3}{2} V_{\max } \\
& =\sum_{j=j_{0}}^{j_{\max }} \int_{x_{j}-\Delta x / 2}^{x_{j}+\Delta x / 2} J(z) E\left(v_{i+j}-v_{i}\right) d z-\frac{3}{2} V_{\max } \\
& =\sum_{j=j_{0}}^{j_{\max }} \mathrm{E}\left(v_{i+j}-v_{i}\right) \int_{x_{j}-\Delta x / 2}^{x_{j}+\Delta x / 2} J(z) d z-\frac{3}{2} V_{\max } \\
& =\sum_{j=j_{0}}^{j_{\max }} \mathrm{E}\left(v_{i+j}-v_{i}\right) J_{j}-\frac{3}{2} V_{\max },
\end{aligned}
$$

with $\mathrm{J}_{j}=\mathrm{V}\left(\mathrm{x}_{\mathrm{j}}+\Delta \mathrm{x} / 2\right)-\mathrm{V}\left(\mathrm{x}_{\mathrm{j}}-\Delta \mathrm{x} / 2\right)$.

Remark 22. Recall that we want to obtain a numerical approximation of the constant called flux-limiter $\bar{A}$ in order for the macroscopic model (5) to be completely determined. Moreover, we recall that $\bar{A}$ contains the memory of the effect of the microscopic perturbation (function $\phi$ in (1)). In the numerical tests we study the influence of the form of the local perturbation (function $\phi$ ) but also we study the influence of the parameters $l, \delta$ and $\mathcal{R}$ from (9) on the approximation of $\bar{A}$. Moreover, the previous algorithm 
provides an approximation of the solution of (9) but given that the goal of this article is to approximate $\bar{A}$ we only use one point provided by the algorithm but an adaptation of this algorithm could be used to approximate solutions of non-local non-linear PDEs.

\subsection{Qualitative properties of $\bar{A}$}

Before passing to the numerical tests, we recall a final result from Forcadel et al. [13] regarding the qualitative properties of $\bar{A}$, which we numerically verify in the next section.

Proposition 23 (Qualitative properties of the flux limiter). Given Assumption 1, the following qualitative properties on the flux limiter hold.

1. (Monotonicity of the flux-limiter) Let $\phi_{1}, \phi_{2}: \mathbb{R} \rightarrow[0,1]$ be two functions satisfying (A6). Let $\overline{\mathrm{A}}_{1}$ and $\overline{\mathrm{A}}_{2}$ be their respective flux limiters. If, for all $x \in \mathbb{R}, \phi_{1}(x) \leqslant \phi_{2}(x)$, then $\bar{A}_{1} \geqslant \bar{A}_{2}$.

2. (Flux interruption) Let $\phi$ be a function satisfying (A6). If $\phi=0$ on an open interval, then $\overline{\mathrm{A}}=0$.

\subsection{Numerical tests}

\section{Influence of $\phi_{0}$}

First, we numerically verify Proposition 23.1 and see the influence of $\phi_{0}=$ $\min _{x} \phi(x)$. We consider a Greenshields optimal velocity function,

$$
V(h)= \begin{cases}0 & \text { if } h \leqslant h_{0} \\ V_{\max }\left[1-\left(h_{0} / h\right)^{2}\right] & \text { if } h>h_{0} \\ V_{\max }\left[1-\left(h_{0} / h_{\max }\right)^{2}\right] & \text { if } h>h_{\max }\end{cases}
$$


For the perturbation, we consider a function

$$
\phi(x)= \begin{cases}1 & \text { if }|x| \geqslant r \\ \phi_{0} & \text { if }|x| \leqslant r / 8 \\ -\frac{8 x\left(1-\phi_{0}\right)}{7 r}+\frac{8 \phi_{0}-1}{7} & \text { if } x \in(-r,-r / 8), \\ \frac{8 x\left(1-\phi_{0}\right)}{7 r}+\frac{8 \phi_{0}-1}{7} & \text { if } x \in(r / 8, r) .\end{cases}
$$

For the values of the different parameters for the optimal velocity function, we take

$$
\left\{\begin{array}{l}
\mathrm{V}_{\max }=58 \mathrm{~km} / \mathrm{h}, \\
\mathrm{h}_{0}=2 \mathrm{~m}, \\
\mathrm{~h}_{\max }=25 \mathrm{~m} .
\end{array}\right.
$$

For the local perturbation, we consider the radius of the perturbation $r=45 \mathrm{~m}$. Figure 3 shows an example of the local perturbation for two different values of $\phi_{0}$. Notice that given the definition (24), if we consider two functions $\phi^{1}$, $\phi^{2}$ with their respective minima $\phi_{0}^{1}<\phi_{0}^{2}$, then $\phi^{1}(x) \leqslant \phi^{2}(x)$ for all $x \in \mathbb{R}$.

In order to see the influence of $\phi_{0} \in[0,1]$ on $\bar{A}$, we discretize the interval $[0,1] \ni \phi_{0}$ in 21 points (a step of 0.05 ) and we compute our estimate of $\bar{A}$ for each of those $\phi_{0}$. For each $\phi_{0}$, we plot two points, since we have an interval that approximates $\bar{A}$.

Figure 4 shows that the approximation is decreasing with $\phi_{0}$, which numerically confirms Proposition 23.1 and notice that for $\phi_{0}=0, \bar{A}$ is close to 0 which numerically confirms Proposition 23.2.

In the case $\phi_{0}=1$, the model is equivalent to a model without a perturbation. Therefore, we should not have a flux-limiting condition. Given the definition (6) of $F_{\bar{A}}$ this can only happen if $\bar{A}=\min _{p} \bar{H}(p)$. In our computational setting $\mathrm{H}_{0} \approx-11.16$ and the approximation of the flux-limiter $\bar{A}$ is of -11.11 which is not very far and which also validates our numerical approach. 
Figure 3: Example of $\phi$ with $r=45 \mathrm{~m}$ : (red) $\phi_{0}=0.25$; and (green) $\phi_{0}=0.5$.

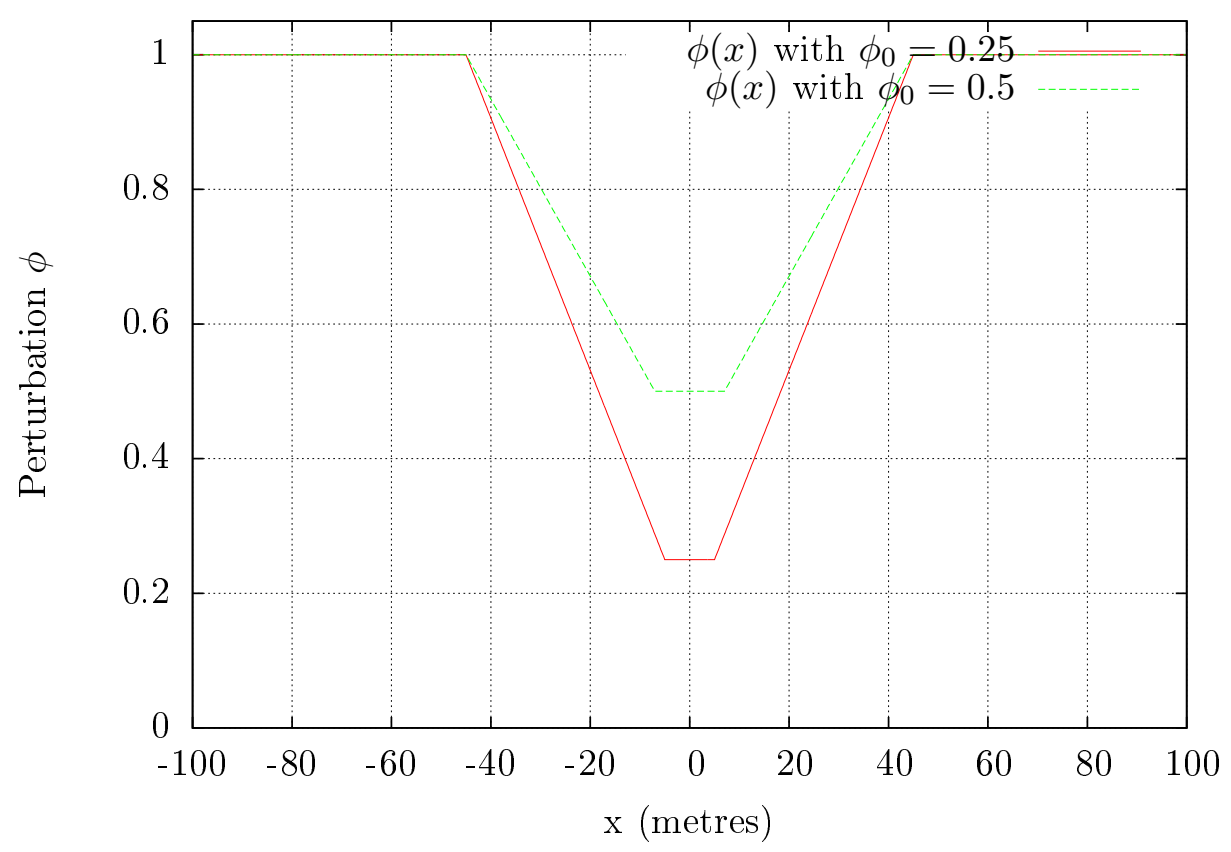

\section{Influence of the radius of influence of the perturbation}

We consider the same optimal velocity function as in the previous simulation and the same perturbation and we make the radius of influence of the perturbation vary in the interval $[25,75]$. Figure 5 contains the approximation of $\bar{A}$ for $\phi_{0}=0.25$ and for different values of $r \in[25,75]$.

Figure 5 shows that in this case the approximation of $\bar{A}$ increases with the radius of the perturbation. However, for $r \geqslant 40 \mathrm{~m}$ the approximation remains the same which could imply that for $r$ big enough, the radius of the perturbation does not influence the value of $\bar{A}$. Moreover, for smaller values of $r, \bar{A}$ is smaller (meaning that the flux is less limited) which is logical, since for a radius $r=0$, we expect to have $\bar{A}=H_{0}$ (there is no perturbation). 
Figure 4: Approximation of $\overline{\mathrm{A}} \in\left[-\delta v_{0}^{+},-\delta \mathrm{u}_{0}^{-}\right]$plotted versus different values of $\phi_{0}$. The curves $-\delta v_{0}^{+}$(in red) and $-\delta u_{0}^{-}$(in green) were computed using Section 6.1 for different values of $\phi_{0}$.

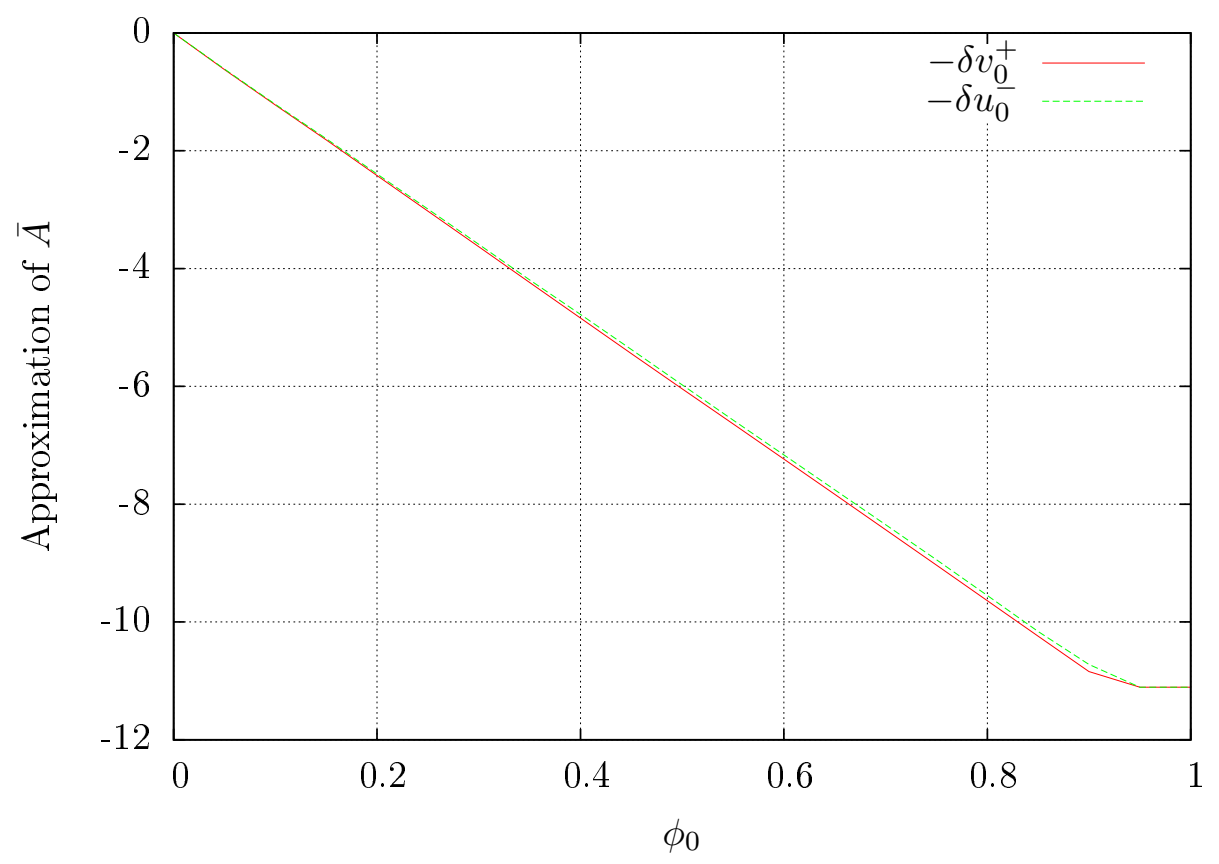

\section{Two different perturbations}

Now we take into account two different perturbations and see how does our approximation of $\bar{A}$ changes with each perturbation. We consider the same perturbation as in the previous simulation and we introduce the following perturbation

$$
\tilde{\phi}(x)= \begin{cases}1 & \text { if }|x| \geqslant r, \\ \frac{\left(1-\phi_{0}\right) x^{2}}{r^{2}}+\phi_{0} & \text { if }|x| \leqslant r .\end{cases}
$$

We consider the same radius of influence $r=45 \mathrm{~m}$. Figure 6 plots the two perturbations $\phi$ and $\tilde{\phi}$ with $\phi_{0}=0.25$. 
Figure 5: Approximation of $\overline{\mathrm{A}} \in\left[-\delta v_{0}^{+},-\delta \mathrm{u}_{0}^{-}\right]$plotted versus different values of $r$ and $\phi_{0}=0.25$. The curves $-\delta v_{0}^{+}$(in red) and $-\delta u_{0}^{-}$(in green) were computed using Section 6.1 for different values of $r$.

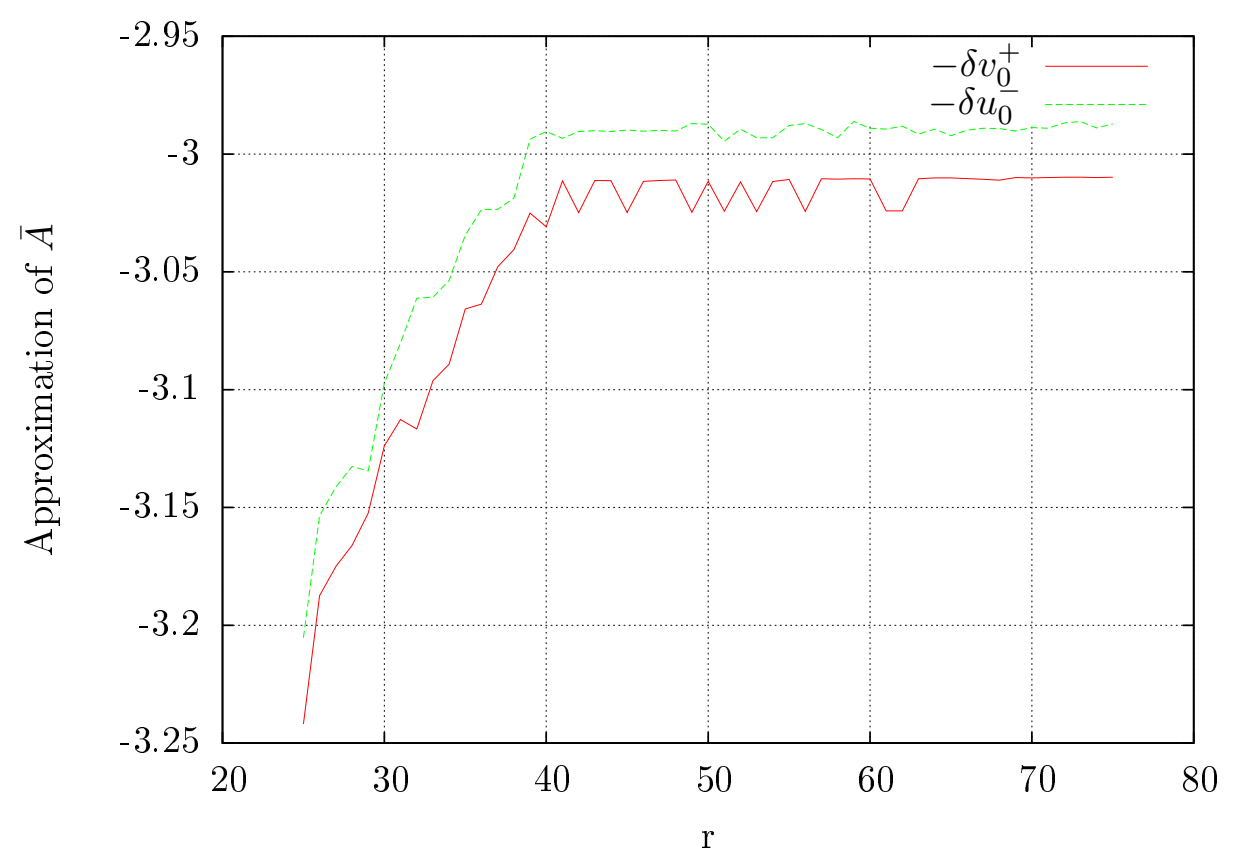

As before, we make $\phi_{0}$ vary inside the interval $[0,1]$. Figure 7 compares the upper and lower bound of the approximation of $\bar{A}$ for different values of $\phi_{0}$.

Figure 7 shows that the approximation of $\bar{A}$ for $\tilde{\phi}$ numerically verifies Proposition 23. Moreover, notice that the values for both perturbations are very similar. This could imply that the form of the perturbation does not influence the result but it is only $\phi_{0}$ that determines the value of the flux limiter. Figure 8 plots the absolute difference between the two approximations and we notice the difference is very small. 
Figure 6: The functions $\phi$ and $\tilde{\phi}$, with $r=45 \mathrm{~m}$ and $\phi_{0}=0.25$.

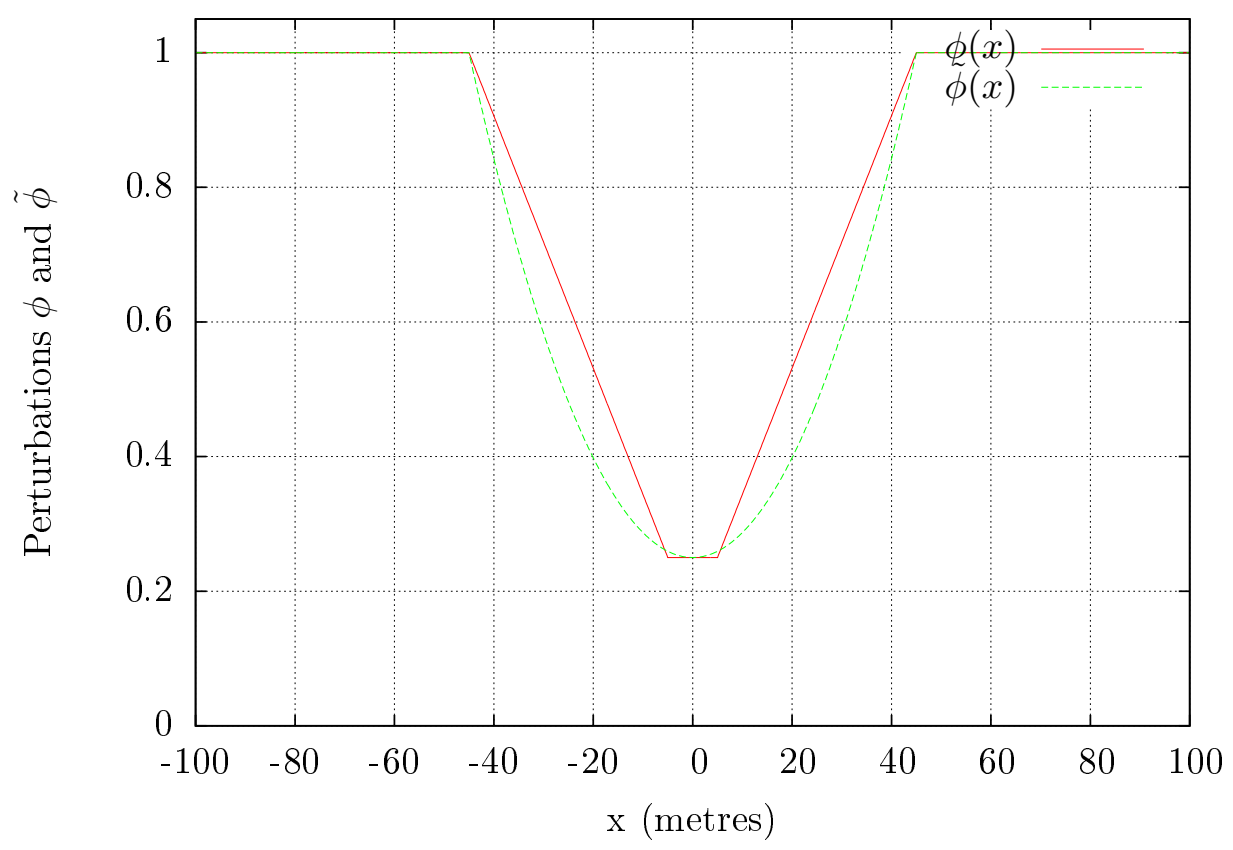

\section{Influence of $\delta$}

We consider the optimal velocity function (23) with (25) and the perturbation (24), with $r=45 \mathrm{~m}$ and $\phi_{0}=0.25$. To see the influence of $\delta$ on the approximation of $\bar{A}$, we fix $l$ and $R$ to the values of (22) and we make $\delta$ vary in $[0.001,0.1]$ with a step of 0.001 .

Figure 9 shows that there is a lot of oscillations on the behaviour of the estimates of $\bar{A}$. However, the upper and lower bound remain close to each other. Given that the difference between the estimates is small, we can assume that considering $\delta=0.001$ gives a good enough approximation of the flux-limiter. 
Figure 7: Lower bound for the approximation of $\bar{A}$ for $\phi$ and $\tilde{\phi}$ versus different values of $\phi_{0}$ (left) upper bound for the approximation of $\bar{A}$ for $\phi$ and $\tilde{\phi}$ versus different values of $\phi_{0}$ (right).
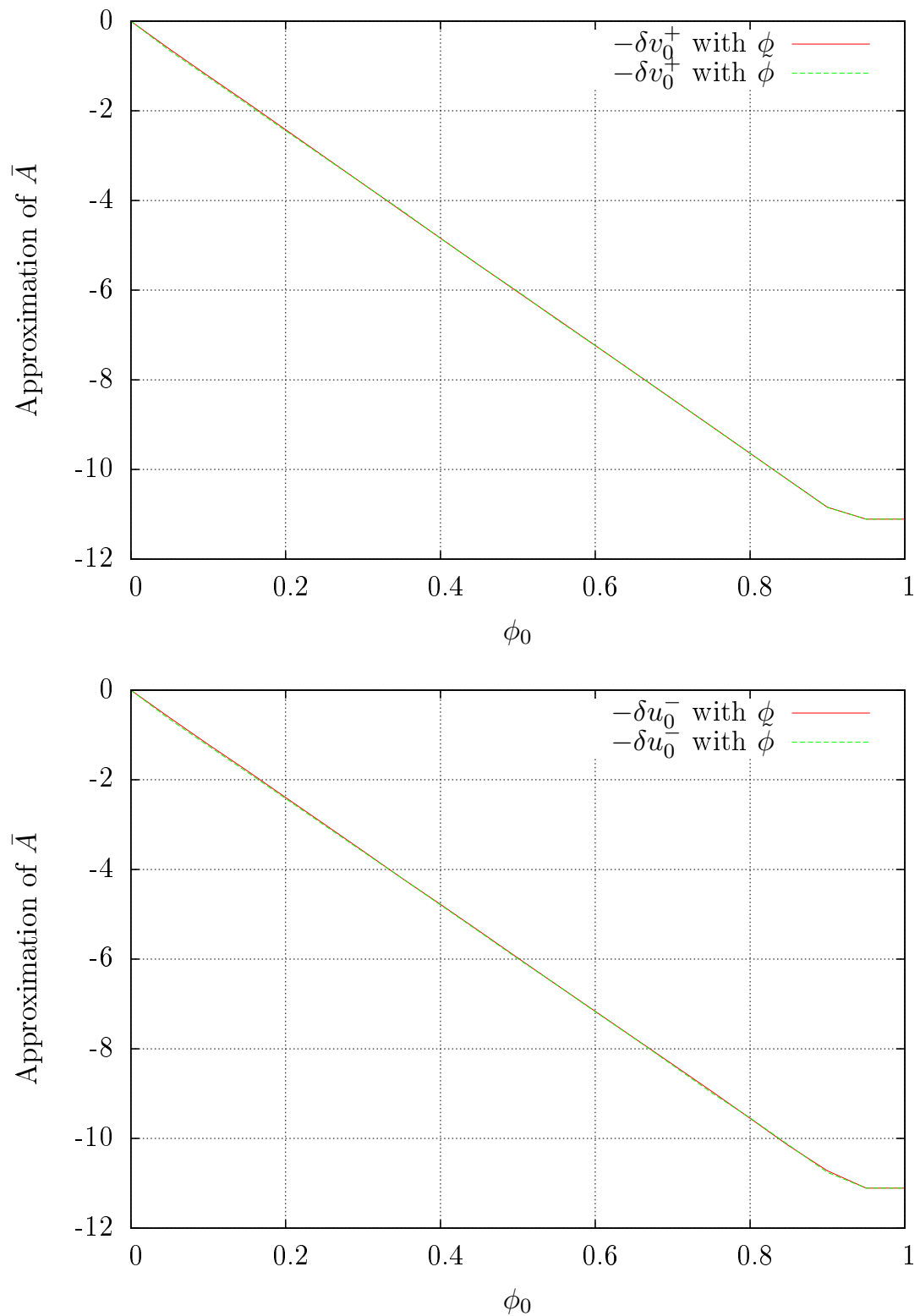
Figure 8: Absolute difference of the lower bound for the approximation of $\bar{A}$ for $\phi$ and $\tilde{\phi}$ versus different values of $\phi_{0}$ (left). Absolute difference of the upper bound for the approximation of $\bar{A}$ for $\phi$ and $\tilde{\phi}$ versus different values of $\phi_{0}$ (right).
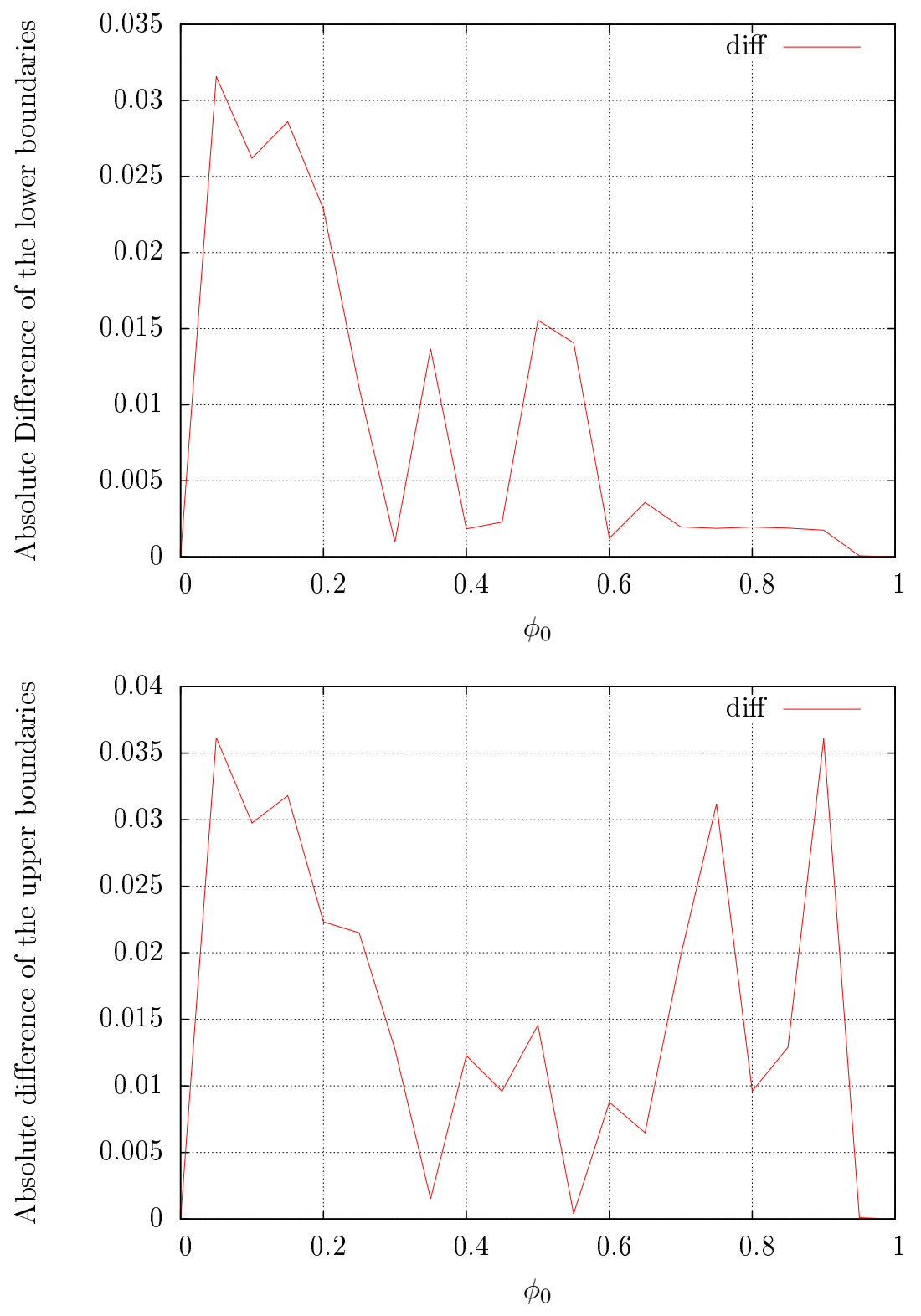
Figure 9: Approximation of $\overline{\mathrm{A}} \in\left[-\delta v_{0}^{+},-\delta \mathrm{u}_{0}^{-}\right]$versus different values of $\delta$. The curves $-\delta v_{0}^{+}$(in red) and $-\delta u_{0}^{-}$(in green) were computed using Section 6.1 for different values of $\delta$.

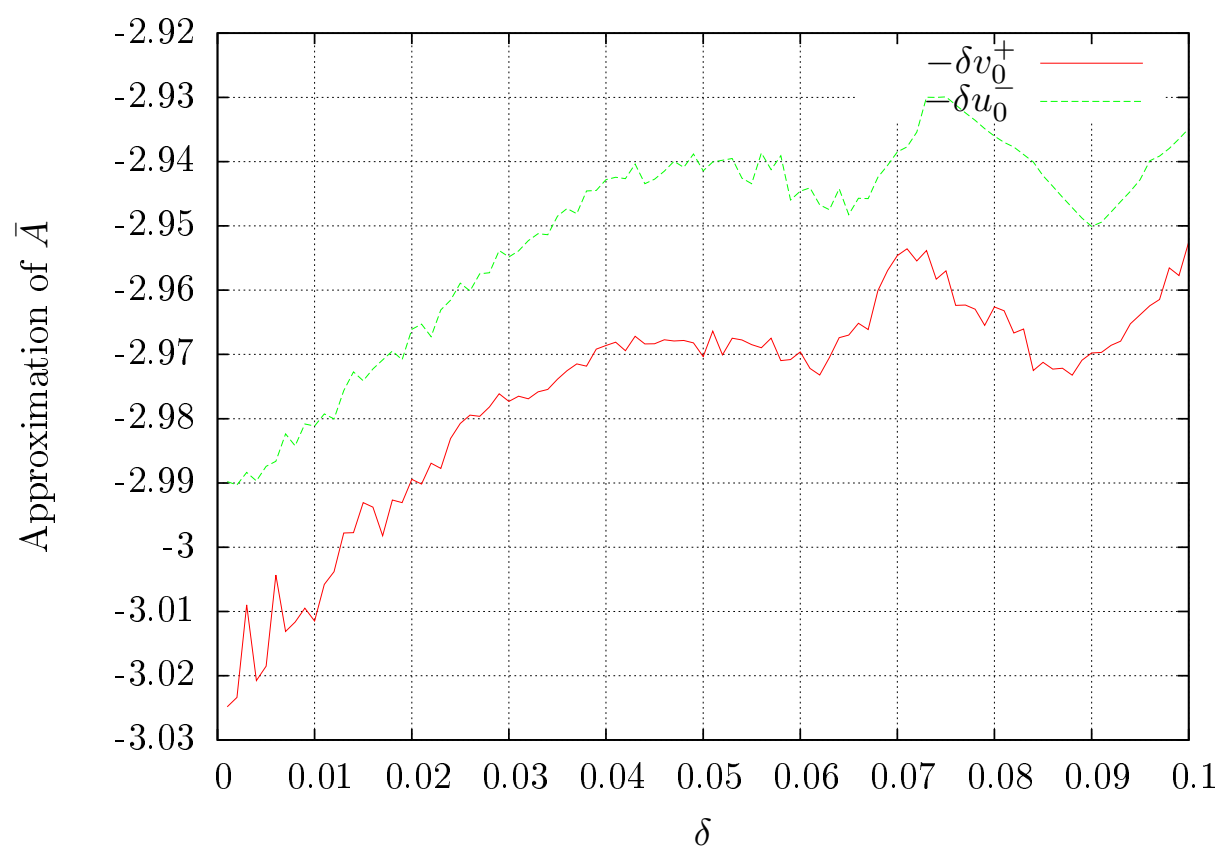

\section{Influence of R (transition between the non-local and local operators)}

We consider the same optimal velocity function and perturbation as in the previous simulation, with the same parameters. To see the influence of $R$ on the approximation of $\bar{A}$, we fix $l$ and $\delta$ to the values of (22) and we make $R$ vary in $[80,150]$ with a step of 1.

Figure 10 shows a lot a oscillation on the behaviour of the estimates for $\bar{A}$ when we make $R$ vary. However, notice that for $R>80$ the difference between the upper and lower estimate is very small (less than 0.4). This suggest that 
Figure 10: Approximation of $\overline{\mathrm{A}} \in\left[-\delta v_{0}^{+},-\delta \mathrm{u}_{0}^{-}\right]$versus different values of R. The curves $-\delta v_{0}^{+}$(in red) and $-\delta u_{0}^{-}$(in green) were computed using Section 6.1 for different values of $R$.

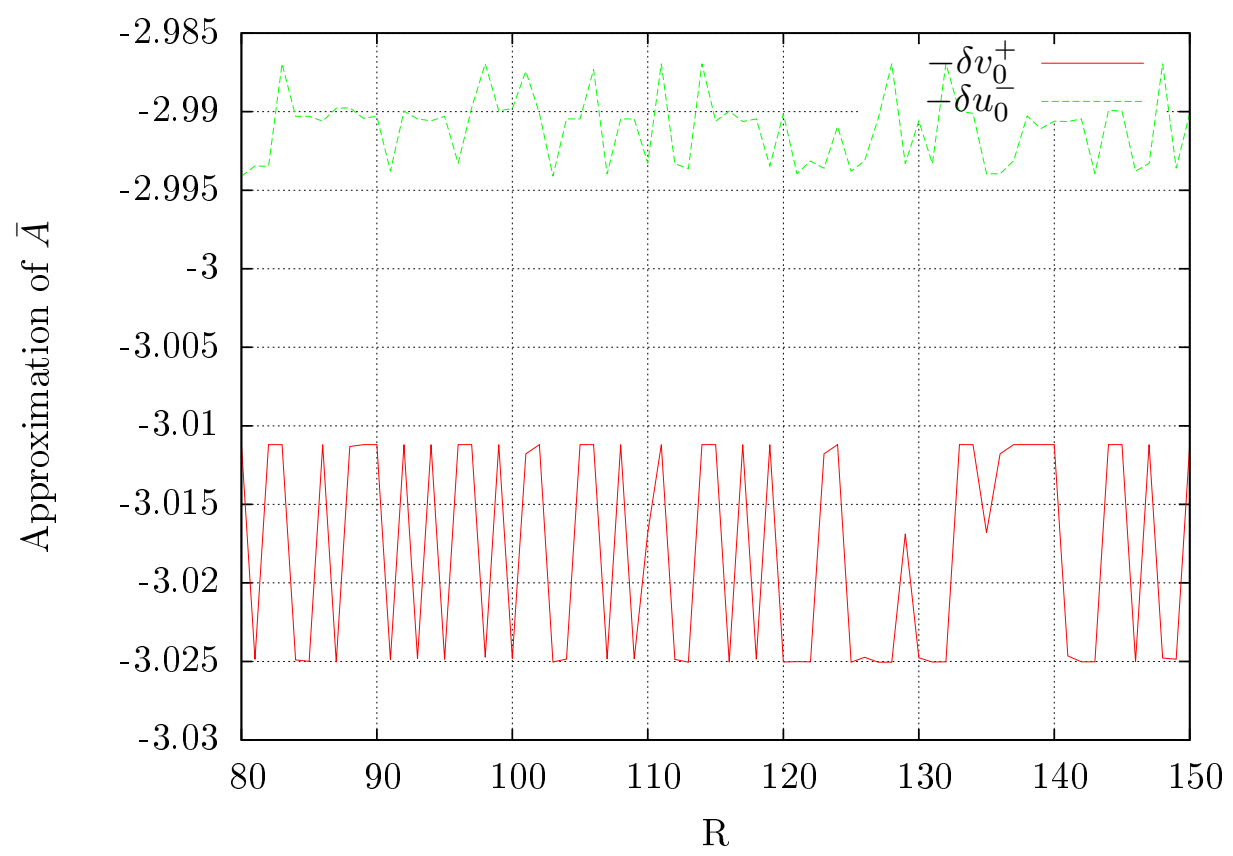

considering $R>80$ is enough for a good approximation of $\bar{A}$.

\section{Influence of $l$}

We consider the same optimal velocity function and perturbation as in the previous simulation, with the same parameters. To see the influence of $l$ on the approximation of $\bar{A}$, we fix $R$ and $\delta$ to the values of (22) and we make $l$ vary in $[180,300]$ with a step of 1.

Figure 11 shows that for $l \in[180,220]$ the approximation of $\bar{A}$ remains 
Figure 11: Approximation of $\overline{\mathrm{A}} \in\left[-\delta v_{0}^{+},-\delta \mathrm{u}_{0}^{-}\right]$versus different values of $l$. The curves $-\delta v_{0}^{+}$(in red) and $-\delta u_{0}^{-}$(in green) were computed using Section 6.1 for different values of $l$.

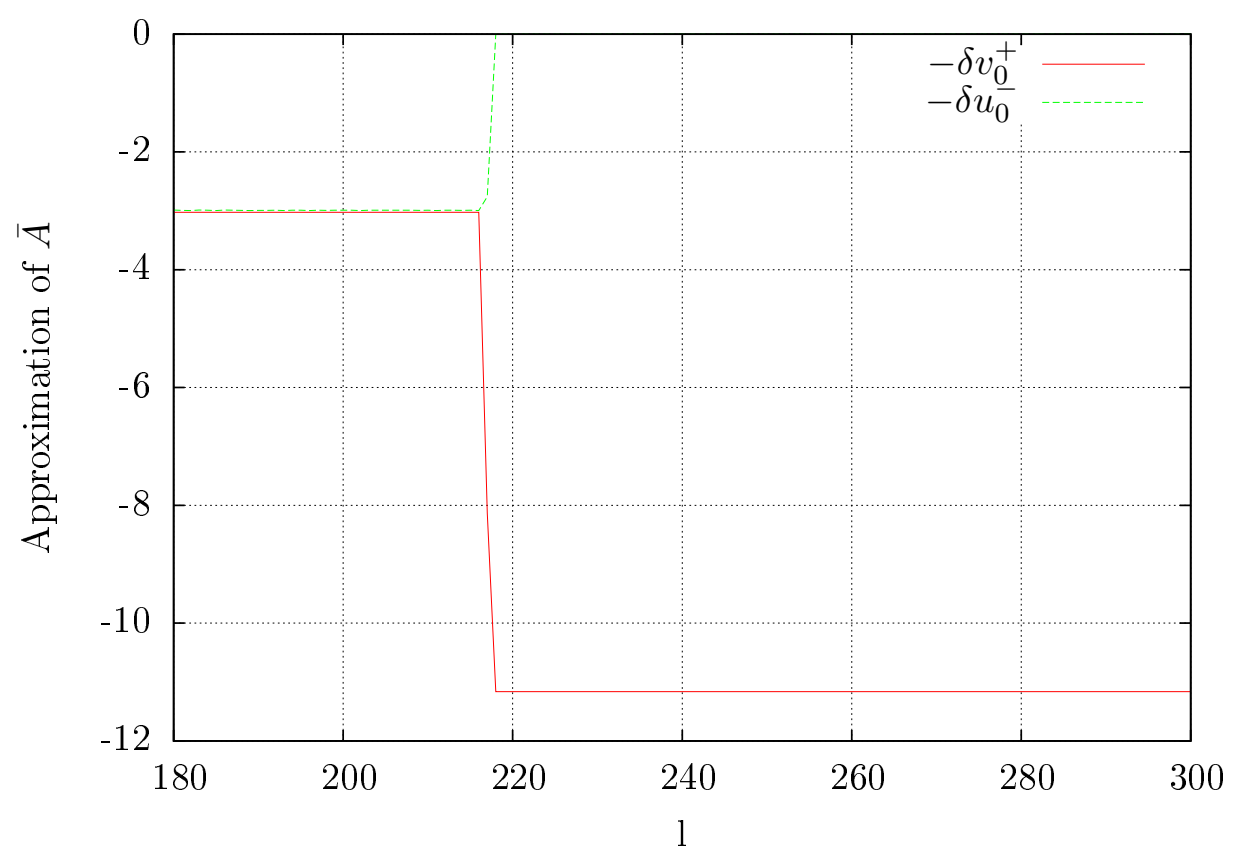

constant. The behaviour of the approximation for bigger values of $l$ can be explain by the fact that first $\delta$ should go to 0 before passing to the limit as $l$ goes to infinity. Therefore, there is a compromise to be made between $l$ and $\delta$. However, for $\delta=0.001$ taking $l=200$ seems to give a reasonable approximation.

\subsection{Conclusion}

We have completed the result previously presented by Forcadel, Salazar and Zaydan [13] by providing a numerical approximation of the constant called 
flux limiter $\bar{A}$. We recall that this constant contains at the macroscopic scale the memory of a local perturbation that slows down vehicles at the microscopic scale. In order to obtain such an approximation we were forced to use equation (9) that was obtain by Forcadel et al. [13] while proving the homogenisation result in that article for the microscopic model (1).

Equation (9) is a non-local non-linear equation which solution we needed in order to approximate the flux limiter $\bar{A}$. However, the form of this PDE allowed us to use very interesting numerical analysis techniques. We used a numerical scheme inspired by the ones of Cacace et al. [6], Costeseque et al. [7] and Forcadel [11] (used for traffic flow and dislocations dynamics). The techniques we use can be used to discretise non-local operators and non-linear terms. Moreover, we provided a convergence result to justify our numerical scheme. The proof of convergence is an adaptation for non-local PDEs of the proof for numerical scheme for non-linear PDEs done by Barles and Souganidis [4].

Moreover, we presented an algorithm to compute an interval that contains an approximation of $\boldsymbol{v}^{\delta}(\boldsymbol{x})$ solution of (9) at the point $\boldsymbol{x}$. This approximation allows us to compute the approximation of the flux limiter. This algorithm inspired by the one by Cacace et al. [6] (used for dislocations dynamics) and justified by the results in Appendix A can be adapted for other PDEs given that the numerical scheme satisfies similar conditions to the ones we have in our scenario.

In the last section, the numerical results allowed us to numerically verify some of the qualitative properties of $\bar{A}$ proven by Forcadel et al. [13] which justifies our choice of numerical scheme. These results also allowed us to see the influence of the different parameters of (9) on the approximation of the flux limiter.

Obtaining macroscopic models for traffic flow like (5) from microscopic models is very important because it allows us to see at the macroscopic scale the influence of microscopic details. This allows us to rigorously obtain more precise macroscopic models. 


\section{A Discrete approximated cell problem}

The following results are similar to the ones by Cacace et al. [6], for the readers convenience, we recall them and adapt them to our problem. The idea is to obtain some results on the numerical scheme to construct a solution to $(12)$.

\section{A.1 Comparisons for the numerical scheme}

In this section, we prove the existence of solutions for the numerical scheme (12). We use the following notations, for every $s \in \mathbb{R}$ and every discrete function $v$, we define

$$
\mathrm{D}_{v_{i}}^{+}(s)=\frac{v_{i+1}-s}{\Delta x}, \quad D_{v_{i}}^{-}(s)=\frac{s-v_{i-1}}{\Delta x}, \quad D_{v_{i}}(s)=\left(D_{v_{i}}^{-}(s), D_{v_{i}}^{+}(s)\right) .
$$

For every $i \in\{-\mathfrak{n}, \ldots, \mathfrak{n}\}$, we set

$$
\begin{aligned}
M_{i}^{\mathrm{d}}[v](s)= & \int_{h_{0}}^{h_{\max }} \mathrm{J}(z) E\left(v_{\sharp}\left(x_{i}+z\right)-s\right) \mathrm{d} z-\frac{3}{2} V_{\max }, \\
\tilde{M}_{i}^{\mathrm{d}}[v](s)= & \int_{h_{0}}^{h_{\max }} \mathrm{J}(z) \tilde{E}\left(v_{\sharp}\left(x_{i}+z\right)-s\right) \mathrm{d} z-\frac{3}{2} V_{\max }, \\
\mathcal{R}_{i}[v](s)= & \psi_{\mathrm{R}}\left(x_{i}\right) \cdot \phi\left(x_{i}\right) \cdot M_{i}^{\mathrm{d}}[v](s) \cdot \mathrm{G}^{+}\left(D_{v_{i}}(s)\right) \\
& +\left(1-\psi_{\mathrm{R}}\left(x_{i}\right)\right) \cdot \bar{H}^{\mathrm{d}}\left(D_{v_{i}}(s)\right) .
\end{aligned}
$$

We define similarly $\tilde{\mathcal{R}}_{i}$ by replacing $M_{i}^{\mathrm{d}}$ with $\tilde{M}_{i}^{\mathrm{d}}$. Finally, we define for all $i \in\{-\mathfrak{n}, \ldots, n\}$,

$$
F_{i}[v](s)= \begin{cases}\mathcal{R}_{\mathfrak{i}}[v](s) & \text { if } i \in\{-\mathrm{n}+1, \ldots, n-1\} \\ \bar{H}^{-}\left(D_{v_{i}}^{+}(s)\right) & \text { if } i=-n \\ \bar{H}^{+}\left(D_{v_{i}}^{-}(s)\right) & \text { if } i=n\end{cases}
$$

Similarly we define $\tilde{\mathrm{F}}_{i}$ by replacing $\mathcal{R}_{\mathfrak{i}}$ by $\tilde{\mathcal{R}}_{\mathfrak{i}}$. 
Remark 24 (Monotonicity of the numerical scheme). By the definition of $F_{i}$ and $\tilde{F}_{\boldsymbol{i}}$ and by Lemma 18, for all $\boldsymbol{i} \in\{-\mathfrak{n}, \ldots, \mathfrak{n}\}$ :

1. $F_{i}[v](s)$ and $\tilde{F}_{i}[v](s)$ are non-decreasing with respect to $s ;$

2. $F_{i}[v](s)$ and $\tilde{F}_{i}[v](s)$ are non-increasing with respect to $v$;

3. $\mathrm{F}_{i}[v]\left(v_{i}\right)=\mathrm{F}_{i}\left([v], \mathrm{D} v_{i}\right)$ and $\tilde{\mathrm{F}}_{i}[v]\left(v_{i}\right)=\tilde{\mathrm{F}}_{i}\left([v], \mathrm{D} v_{i}\right)$;

4. $\tilde{F}_{i}[v](s) \leqslant F_{i}[v](t)$ for all $s<t$.

Lemma 25 (Comparison). Let $\boldsymbol{v}$ be a discrete function and let $\mathbf{i} \in\{-\mathfrak{n}, \ldots, \mathfrak{n}\}$. There exists a unique $s_{i} \in \mathbb{R}$ such that

$$
-\tilde{F}_{i}[v]\left(s_{i}\right) \leqslant \delta s_{i} \leqslant-F_{i}[v]\left(s_{i}\right) .
$$

Moreover, let $w$ be a discrete function. Then the following implications hold:

1. $\delta w_{i}+F_{i}[w]\left(w_{i}\right) \leqslant 0$ and $w \leqslant v \Rightarrow w_{i} \leqslant s_{i} ;$

2. $\delta w_{i}+F_{i}[w]\left(w_{i}\right)>0$ and $w \geqslant v \Rightarrow w_{i}>s_{i}$;

3. $\delta w_{i}+\tilde{F}_{i}[w]\left(w_{i}\right) \geqslant 0$ and $w \geqslant v \Rightarrow w_{i} \geqslant s_{i}$;

4. $\delta w_{i}+\tilde{F}_{i}[w]\left(w_{i}\right)<0$ and $w \leqslant v \Rightarrow w_{i}<s_{i}$.

Proof: The existence of a $s_{i} \in \mathbb{R}$ satisfying (26) comes from $-F_{i}[v](s)$ and $-\tilde{F}_{i}[v](s)$ are non-increasing in $s$ and that $\delta s$ is strictly increasing in $s$. As for the uniqueness of $s_{i}$, let us argue by contradiction and assume there exists $s_{i}^{1}$ and $s_{i}^{2}$ with $s_{i}^{1}<s_{i}^{2}$ such that $-\tilde{F}_{i}[v]\left(s_{i}^{1}\right) \leqslant \delta s_{i}^{1} \leqslant-F_{i}[v]\left(s_{i}^{1}\right)$ and $-\tilde{F}_{i}[v]\left(s_{i}^{2}\right) \leqslant \delta s_{i}^{2} \leqslant-F_{i}[v]\left(s_{i}^{2}\right)$. However, using Remark $24.4,-F_{i}[v]\left(s_{i}^{2}\right) \leqslant$ $-\tilde{F}_{i}[v]\left(s_{i}^{1}\right)$, using this and combining the previous inequalities we obtain

$$
F_{i}[v]\left(s_{i}^{1}\right)-\tilde{F}_{i}[v]\left(s_{i}^{2}\right) \leqslant \delta\left(s_{i}^{2}-s_{i}^{1}\right) \leqslant \tilde{F}_{i}[v]\left(s_{i}^{1}\right)-F_{i}[v]\left(s_{i}^{2}\right) \leqslant 0 .
$$

This gives us that $s_{2}-s_{1} \leqslant 0$ which is a contradiction. 
We now prove Lemma 25.1, again we argue by contradiction and assume that $w_{i}>s_{i}$. Then using (26),

$$
\delta w_{i}>\delta s_{i} \geqslant-\tilde{F}_{i}[v]\left(s_{i}\right) \geqslant-\tilde{F}_{i}[w]\left(s_{i}\right) \geqslant-F_{i}[w]\left(w_{i}\right) \geqslant \delta w_{i}
$$

where we have used for the third and fourth inequality respectively Remark 24.2 and Remark 24.4. The previous inequality gives us the desired contradiction.

We now prove Lemma 25.2. We argue by contradiction and assume that $w_{i} \leqslant s_{i}$, using (26),

$$
\delta w_{i} \leqslant \delta s_{i} \leqslant-F_{i}[v]\left(s_{i}\right) \leqslant-F_{i}[w]\left(s_{i}\right) \leqslant-F_{i}[w]\left(w_{i}\right)<\delta w_{i},
$$

where we have used for the third and fourth inequality respectivelyRemark 24.1 and Remark 24.2. The previous inequality gives us the desired contradiction. The proofs of Lemma 25.3 and Lemma 25.4 are similar and we skip them.

\section{A.2 Construction of minimal and maximal solutions}

This section is devoted to the proof of existence and to the construction of minimal and maximal solutions. In order to prove the existence of discrete solution for (12), we provide a constructive method that provides us with the minimal and maximal solutions.

Proposition 26 (Definition of the map $\Phi$ ). There exists a map $\Phi: \mathbb{R}^{2 n+1} \rightarrow$ $\mathbb{R}^{2 \mathfrak{n}+1}$ satisfying the following properties.

1. Let $\mathfrak{u}^{-}$be a sub-solution of $(12)$, that is, $\delta \mathbf{u}_{\mathfrak{i}}^{-}+\mathrm{F}_{\mathfrak{i}}\left[\mathrm{u}^{-}\right]\left(\mathrm{u}_{\mathfrak{i}}^{-}\right) \leqslant 0$ for all $\mathfrak{i} \in\{-\mathfrak{n}, \ldots, \mathfrak{n}\}$. Then

(a) $\mathrm{u}^{-} \leqslant \Phi\left[\mathrm{u}^{-}\right]$(with $\mathfrak{u}^{-}=\Phi\left[\mathrm{u}^{-}\right]$if and only if $\mathfrak{u}^{-}$is a solution);

(b) $\Phi\left[\mathrm{u}^{-}\right]$is a sub-solution of (12). 
2. Let $\mathfrak{u}^{+}$be a super-solution of (12), that is, $\delta u_{i}^{-}+\tilde{F}_{i}\left[u^{-}\right]\left(u_{i}^{-}\right) \geqslant 0$ for all $\boldsymbol{i} \in\{-\mathfrak{n}, \ldots, \mathfrak{n}\}$. Then
(a) $\mathfrak{u}^{+} \geqslant \Phi\left[\mathfrak{u}^{+}\right]$(with $\mathbf{u}^{+}=\Phi\left[\mathfrak{u}^{+}\right]$if and only if $\mathfrak{u}^{+}$is a solution);
(b) $\Phi\left[\mathfrak{u}^{+}\right]$is a super-solution of (12).

Proof: Let us prove the result for the sub-solutions Proposition 26.1. Using Lemma 25, for every $i \in\{-\mathfrak{n}, \ldots, \mathfrak{n}\}$ there exists a unique $\boldsymbol{s}_{\boldsymbol{i}}^{-} \in \mathbb{R}$ such that

$$
-\tilde{F}_{i}\left[u^{-}\right]\left(s_{i}^{-}\right) \leqslant \delta s_{i}^{-} \leqslant-F_{i}\left[u^{-}\right]\left(s_{i}^{-}\right) \text {and } u_{i}^{-} \leqslant s_{i}^{-} \text {, }
$$

the second inequality comes from Lemma 25.1 considering $w=\mathfrak{u}^{-}$. Using the real numbers $s_{i}^{-}$we construct the map $\Phi\left[\mathfrak{u}^{-}\right]_{i}=s_{i}^{-}$for all $i \in\{-n, \ldots, n\}$. By construction $\mathfrak{u}^{-} \leqslant \Phi\left[\mathfrak{u}^{-}\right]$, with the equality if and only if $\mathfrak{u}^{-}$is a solution of (12) (which proves (i) in (a)). Let us now prove that $\Phi\left[\mathbf{u}^{-}\right]$is a sub-solution of (12). For all $i \in\{-\mathfrak{n}, \ldots, \mathfrak{n}\}$, by the definition of $s_{\mathfrak{i}}^{-}$,

$$
\begin{aligned}
0 \geqslant \delta s_{i}^{-}+F_{i}\left[u^{-}\right]\left(s_{i}^{-}\right) \geqslant & \delta s_{i}^{-}+F_{i}\left[\Phi\left[u^{-}\right]\right]\left(s_{i}^{-}\right) \\
& =\delta \Phi\left[u^{-}\right]_{i}+F_{i}\left[\Phi\left[u^{-}\right]\right]\left(\Phi\left[u^{-}\right]_{i}\right),
\end{aligned}
$$

where we use the second inequality that $F_{i}[\cdot](s)$ is non-increasing and that $\mathfrak{u}^{-} \leqslant \Phi\left[\mathfrak{u}^{-}\right]$. Therefore, $\Phi\left[\mathfrak{u}^{-}\right]$is a sub-solution of (12).

In the case of the super-solutions, Proposition 26.2, we define the map $\Phi$ in the same way. Using Lemma 25 , for every $i \in\{-\mathfrak{n}, \ldots, n\}$ there exists a unique $s_{i}^{+} \in \mathbb{R}$ such that

$$
-\tilde{F}_{i}\left[u^{+}\right]\left(s_{i}^{+}\right) \leqslant \delta s_{i}^{+} \leqslant-F_{i}\left[u^{+}\right]\left(s_{i}^{+}\right) \text {and } u_{i}^{+} \geqslant s_{i}^{+} .
$$

As before, the map $\Phi$ is constructed using the real numbers $s_{i}^{+}: \Phi\left[u^{+}\right]_{i}=s_{i}^{+}$ for all $i \in\{-\mathfrak{n}, \ldots, n\}$. Proceeding as in the first part of the proof we can prove the rest of Proposition 26.2. We skip the rest of the proof and this ends the proof of Proposition 26. 
Proposition 27 (Partial comparison principle). Let $\mathfrak{u}^{-}$and $\mathfrak{u}^{+}$be respectively a sub and a super-solution of (12) such that $\mathfrak{u}^{-} \leqslant \mathfrak{u}^{+}$then $\Phi\left[\mathrm{u}^{-}\right] \leqslant \Phi\left[\mathrm{u}^{+}\right]$.

Proof: We argue by contradiction and assume that there exists a $i_{0} \in$ $\{-\mathfrak{n}, \ldots, \mathfrak{n}\}$ such that $\Phi\left[\mathfrak{u}^{-}\right]_{i}=s_{i}^{-}>s_{i}^{+}=\Phi\left[u^{+}\right]_{i}$. By definition of $s_{i}^{+}$,

$$
\begin{aligned}
0 & \leqslant \delta s_{i}^{+}+\tilde{F}_{i}\left[u^{+}\right]\left(s_{i}^{+}\right)<\delta s_{i}^{-}+\tilde{F}_{i}\left[u^{+}\right]\left(s_{i}^{+}\right) \\
& \leqslant \delta s_{i}^{-}+F_{i}\left[u^{+}\right]\left(s_{i}^{-}\right) \leqslant \delta s_{i}^{-}+F_{i}\left[u^{-}\right]\left(s_{i}^{-}\right),
\end{aligned}
$$

where we use that $s_{i}^{-}>s_{i}^{+}$for the second inequality, and Remark 24.1 and Remark 24.2 for the third and fourth inequalities respectively. This inequality gives us the desired contradiction because of the definition of $s_{i}^{-}$.

Proposition 28 (Construction of solutions). Let $\mathbf{u}^{-}$and $\mathbf{u}^{+}$be respectively a sub and a super-solution of (12) such that $\mathfrak{u}^{-} \leqslant \mathfrak{u}^{+}$. We consider for every $\mathrm{k} \in \mathbb{N}, \mathfrak{u}^{-, \mathrm{k}+1}=\Phi\left[\mathrm{u}^{-, \mathrm{k}}\right]$, with $\mathrm{u}^{-, 0}=\mathfrak{u}^{-}$, and $\boldsymbol{v}^{+, \mathrm{k}+1}=\Phi\left[v^{+, \mathrm{k}}\right]$, with $v^{+, 0}=\mathbf{u}^{+}$. There exist two discrete functions $\mathbf{u}$ and $\boldsymbol{v}$ such that $\mathbf{u}^{-, \mathrm{k}} \rightarrow \mathbf{u}$ and $v^{+, k} \rightarrow v$ as $\mathrm{k} \rightarrow+\infty$. Moreover, $\boldsymbol{u}$ and $\boldsymbol{v}$ are two solutions of (12). We define $\Psi\left[\mathrm{u}^{-}\right]:=\mathfrak{u}$ and $\Psi\left[v^{+}\right]:=v$. Then

$$
u^{-} \leqslant \Psi\left[u^{-}\right] \leqslant \Psi\left[u^{+}\right] \leqslant u^{+},
$$

and $\Psi[u]=u$ if and only if $\mathfrak{u}$ is a solution of (12).

Proof: Using Proposition 27 we get the following inequalities

$$
u^{-} \leqslant \Phi\left[u^{-, k}\right] \leqslant \Phi\left[u^{-, k+1}\right] \leqslant \cdots \leqslant \Phi\left[v^{+, k+1}\right] \leqslant \Phi\left[v^{+, k}\right] \leqslant v^{+} .
$$

Therefore, the sequence $\left(u^{-, k}\right)_{k}$ is non-decreasing and bounded from above by $v^{+}$, and the sequence $\left(v^{+, k}\right)_{k}$ is non-increasing and bounded from below 
by $\mathrm{u}^{-}$. Passing to the limit as $\mathrm{k}$ goes to infinity in the previous inequalities, we obtain

$$
\mathfrak{u}^{-} \leqslant \lim _{k \rightarrow+\infty} \mathfrak{u}^{-, k}=: \mathfrak{u}=: \Psi\left[u^{-}\right] \leqslant \Psi\left[v^{+}\right]:=v:=\lim _{k \rightarrow+\infty} v^{+, k} \leqslant v^{+} .
$$

Let us now prove that $u$ is a solution of (12) (the proof for $v$ is similar and we skip it). By definition of the sequence $\left(\mathfrak{u}^{-, k}\right)_{k}$, for all $k \in \mathbb{N}$ and for all $i \in\{-\mathfrak{n}, \ldots, n\}$,

$$
-\tilde{F}_{i}\left[u^{-, k}\right]\left(u_{i}^{-, k+1}\right) \leqslant \delta u_{i}^{-, k+1} \leqslant-F_{i}\left[u^{-, k}\right]\left(u_{i}^{-, k+1}\right) .
$$

We recall that $E$ and $\tilde{E}$ are respectively lower and upper semi-continuous, which implies that $R$ and $\tilde{R}$ are also lower and upper semi-continuous and in particular that $-R$ and $-\tilde{R}$ are respectively upper and lower semi-continuous. Adding this to the continuity of the discrete gradient and of the functions $\overline{\mathrm{H}}$, $\overline{\mathrm{H}}^{+}$and $\overline{\mathrm{H}}^{-}$, passing to the limit as $\mathrm{k}$ goes to $+\infty$ in $(28)$ implies $-\tilde{\mathrm{F}}_{i}[\mathrm{u}]\left(\mathrm{u}_{\mathrm{i}}\right) \leqslant$ $\delta u_{i} \leqslant-F_{i}[u]\left(u_{i}\right)$, which means that $u$ is a solution of (12). Finally, $\Psi[u]=u$ if and only if $u$ is a solution comes from the properties of $\Phi$ (Proposition 26).

Proposition 29 (Extremal solutions in the interval $\left.\left(\mathrm{u}^{-}, v^{+}\right)\right)$. Let $\mathrm{u}^{-}$and $v^{+}$ be respectively a sub and super-solution of (12) such that $\mathfrak{u}^{-} \leqslant v^{+}$. Let $\Psi\left[\mathfrak{u}^{-}\right]$ and $\Psi\left[v^{+}\right]$be the two solutions provided by Proposition 28. Then every solution $\boldsymbol{v}$ of (12) such that $\mathbf{u}^{-} \leqslant v \leqslant v^{+}$satisfies

$$
\mathrm{u}^{-} \leqslant \Psi\left[\mathrm{u}^{-}\right] \leqslant v \leqslant \Psi\left[v^{+}\right] \leqslant v^{+} .
$$

Proof: Considering $v$ as a super-solution of (12), and using Proposition 28, $u^{-} \leqslant \Psi\left[\mathfrak{u}^{-}\right] \leqslant \Psi[v]=v$. Similarly, considering $v$ as a sub-solution of (12), using Proposition $28, v=\Psi[v] \leqslant \Psi\left[v^{+}\right] \leqslant v^{+}$.

Combining Lemma 19 and Propositions 20 and 29 we obtain the following corollary. 
Corollary 30. Let $\mathfrak{u}_{\mathfrak{i}}^{-}=0$ and $v_{i}^{+}=\mathrm{C}_{0} / \delta$ for all $\mathfrak{i} \in\{-\mathfrak{n}, \ldots, \mathfrak{n}\}$. Let $\Psi\left[\mathfrak{u}^{-}\right]$ and $\Psi\left[v^{+}\right]$be the solutions provided by Proposition 28, then every solution $v$ of (12) satisfies $\Psi\left[\mathrm{u}^{-}\right] \leqslant v \leqslant \Psi\left[v^{+}\right]$.

Acknowledgements This project was co-financed by the European Union with the European regional development fund (ERDF, HN0002137) and by the Normandie Regional Council via the M2NUM project and by ANR HJNet (ANR-12-BS01-0008-01). This research used resources of the HighPerformance Computing Centre of Normandy (CRIANN).

\section{References}

[1] Yves Achdou and Nicoletta Tchou. "Hamilton-Jacobi equations on networks as limits of singularly perturbed problems in optimal control: dimension reduction". In: Communications in Partial Differential Equations 40.4 (2015), pp. 652-693 (cit. on p. E59).

[2] Masako Bando et al. "Dynamical model of traffic congestion and numerical simulation". In: Physical Review E 51.2 (1995), p. 1035 (cit. on p. E52).

[3] Guy Barles. Solutions de viscosité des équations de Hamilton-Jacobi. Springer Verlag, 1994 (cit. on p. E64).

[4] Guy Barles and Panagiotis E Souganidis. "Convergence of approximation schemes for fully nonlinear second order equations". In: Asymptotic analysis 4.3 (1991), pp. 271-283 (cit. on pp. E69, E87).

[5] Milan Batista and Elen Twrdy. "Optimal velocity functions for car-following models". In: Journal of Zhejiang University SCIENCE A 11.7 (2010), pp. 520-529 (cit. on pp. E52, E55). 
[6] Simone Cacace, Antonin Chambolle, and Régis Monneau. "A posteriori error estimates for the effective Hamiltonian of dislocation dynamics". In: Numerische Mathematik 121.2 (2012), pp. 281-335 (cit. on pp. E53, E60, E62, E73, E87, E88).

[7] Guillaume Costeseque, Jean-Patrick Lebacque, and Régis Monneau. "A convergent scheme for Hamilton-Jacobi equations on a junction: application to traffic". In: Numerische Mathematik 129.3 (2015), pp. 405-447 (cit. on pp. E53, E60, E62, E87).

[8] Michael G Crandall, Hitoshi Ishii, and Pierre-Louis Lions. "User's guide to viscosity solutions of second order partial differential equations". In: Bulletin of the American Mathematical Society 27.1 (1992), pp. 1-67 (cit. on p. E64).

[9] Francesca Da Lio, Nicolas Forcadel, and Régis Monneau. "Convergence of a non-local eikonal equation to anisotropic mean curvature motion. Application to dislocations dynamics." In: Journal of the European Mathematical Society 10.4 (2008), pp. 1105-1119 (cit. on p. E66).

[10] Leslie C Edie. "Car-following and steady-state theory for noncongested traffic". In: Operations Research 9.1 (1961), pp. 66-76 (cit. on p. E55).

[11] Nicolas Forcadel. "An error estimate for a new scheme for mean curvature motion". In: SIAM journal on numerical analysis 46.5 (2008), pp. 2715-2741 (cit. on pp. E53, E60, E87).

[12] Nicolas Forcadel, Cyril Imbert, and Régis Monneau. "Homogenization of some particle systems with two-body interactions and of the dislocation dynamics". In: Discrete Contin. Dyn. Syst. 23.3 (2009), pp. 785-826. ISSN: 1078-0947. DOI: 10.3934/dcds.2009.23.785. (Cit. on pp. E55, E68).

[13] Nicolas Forcadel, Wilfredo Salazar, and M Zaydan. "A junction condition by specified homogenization of a discrete model with a local perturbation and application to traffic flow". In: <hal-01618395> (2017) (cit. on pp. E52, E53, E55, E56, E57, E59, E60, E66, E69, E76, E86, E87). 
[14] Giulio Galise, Cyril Imbert, and Régis Monneau. "A junction condition by specified homogenization and application to traffic lights". In: Anal. PDE 8.8 (2015), pp. 1891-1929. ISSN: 2157-5045. DOI: 10.2140/apde.2015.8.1891. (Cit. on pp. E59, E68).

[15] Mauro Garavello and Benedetto Piccoli. Traffic flow on networks. American institute of mathematical sciences Springfield, MO, USA, 2006 (cit. on pp. E55, E58).

[16] BD Greenshields, Ws Channing, Hh Miller, et al. "A study of traffic capacity". In: Highway research board proceedings. Vol. 1935. National Research Council (USA), Highway Research Board. 1935 (cit. on p. E54).

[17] Cyril Imbert and Régis Monneau. "Flux-limited solutions for quasi-convex Hamilton-Jacobi equations on networks". In: $<$ hal-00832545> (2014) (cit. on p. E68).

[18] Michael J Lighthill and Gerald Beresford Whitham. "On kinematic waves. II. A theory of traffic flow on long crowded roads". In: Proceedings of the Royal Society of London. Series A. Mathematical and Physical Sciences 229.1178 (1955), pp. 317-345 (cit. on p. E58).

[19] Pierre Louis Lions. Lectures at Collège de France. 2013-2014 (cit. on p. E59).

[20] Gordon Frank Newell. "Nonlinear effects in the dynamics of car following". In: Operations Research 9.2 (1961), pp. 209-229 (cit. on p. E55).

[21] Stanley Osher and James A Sethian. "Fronts propagating with curvature-dependent speed: algorithms based on Hamilton-Jacobi formulations". In: Journal of computational physics 79.1 (1988), pp. 12-49 (cit. on p. E62).

[22] Paul I Richards. "Shock waves on the highway". In: Operations research 4.1 (1956), pp. 42-51 (cit. on p. E58). 
[23] Dejan Slepčev. "Approximation schemes for propagation of fronts with nonlocal velocities and Neumann boundary conditions". In: Nonlinear Analysis: Theory, Methods \&5 Applications 52.1 (2003), pp. 79-115 (cit. on p. E66).

\section{Author address}

1. W. Salazar, Normandie Univ, INSA de Rouen, LMI (EA 3226 - FR CNRS 3335), 76000 Rouen, France, 685 Avenue de l'Université, 76801 St Etienne du Rouvray cedex. France orcid:0000-0002-0419-4071 